\title{
Compositional differences between the component stars of eclipsing close binary systems showing chemical peculiarities
}

\author{
Yoichi Takeda ${ }^{1,2 \star}$, Inwoo Han ${ }^{3,4}$, Dong-Il Kang ${ }^{5}$, Byeong-Cheol Lee ${ }^{3,4}$, Kang-Min Kim³ \\ ${ }^{1}$ National Astronomical Observatory of Japan, 2-21-1 Osawa, Mitaka, Tokyo 181-8588, Japan \\ ${ }^{2}$ SOKENDAI, The Graduate University for Advanced Studies, 2-21-1 Osawa, Mitaka, Tokyo 181-8588, Japan \\ ${ }^{3}$ Korea Astronomy and Space Science Institute, 776, Daedeokdae-ro, Youseong-gu, Daejeon 34055, Korea \\ ${ }^{4}$ Korea University of Science and Technology, 217, Gajeong-ro Youseong-gu, Daejeon 34113, Korea \\ ${ }^{5}$ Changwon Science high school, 30, Pyungsanro 159-th, Uichang, Changwon, 641-500, Korea
}

Accepted 2019 February 9. Received 2019 February 8; in original form 2018 December 3.

\begin{abstract}
A spectroscopic study was carried out on the surface chemical abundances of CNO and several heavier elements in the primary and secondary components of 5 eclipsing close binaries around A-type (AR Aur, $\beta$ Aur, YZ Cas, WW Aur, and RR Lyn), in order to investigate the nature of chemical differences between both components (being comparatively slow rotators alike due to tidal synchronization). Regarding the systems comprising similar components, $\beta$ Aur and WW Aur were confirmed to exhibit no compositional difference between the primary and secondary both showing almost the same Am anomaly, though the chemical peculiarities seen in the component stars of AR Aur show distinct differences (HgMn star and Am star). In contrast, as to the systems (YZ Cas and RR Lyn) consisting of considerably different (A and earlyF) components, the surface abundances are markedly different between the primary (Am) and secondary (normal). These observational results may indicate $T_{\text {eff-dependent }}$ characteristics regarding the chemical anomalies of non-magnetic stars on the upper main sequence: (1) In the effective temperature range of $10000 \mathrm{~K} \gtrsim T_{\text {eff }} \gtrsim 7000 \mathrm{~K}$, rotational velocity is the most important factor for determining the extent of Am peculiarity. (2) However, the emergence of Am phenomenon seems to have a lower $T_{\text {eff }}$ limit at $\sim 7000 \mathrm{~K}$, below which no abundance anomaly is observed regardless of stellar rotation. (3) The transition from Am anomaly (mild deficiency in CNO) to $\mathrm{HgMn}$ anomaly (unusually large $\mathrm{N}$ depletion) is likely to take place as $T_{\text {eff }}$ increases from $\sim 10000 \mathrm{~K}$ to $\sim 11000 \mathrm{~K}$.
\end{abstract}

Key words: stars: abundances — stars: atmospheres — stars: binaries: eclipsing stars: chemically peculiar — stars: early-type

\section{INTRODUCTION}

The nature and origin of chemically peculiar stars (CP stars) seen in late $B$ through early $F$ type stars of upper main sequence, which are divided into several subgroups according to the type of peculiarity (e.g., Preston 1974), has long been a matter of debate. Among these, commonly observed CP phenomena among non-magnetic stars 1 are HgMn stars (mostly seen in late B dwarfs) and Am stars (metallic-lined

\footnotetext{
* E-mail: takeda.yoichi@nao.ac.jp

1 This term "non-magnetic stars" is used to discriminate them from those CP stars showing strong magnetic fields (up to $\sim 10^{3}$ $10^{4}$ gauss) as well as specific abundance anomalies (e.g., overabundances of $\mathrm{Cr}, \mathrm{Sr}$, or $\mathrm{Eu}$ ). Recent high-precision polarimetric
}

A stars, which are widely seen in early-A through early-F main-sequence stars). Takeda et al. (2018c; hereinafter referred to as Paper I) very recently carried out an extensive study of $\mathrm{C}, \mathrm{N}$, and $\mathrm{O}$ abundances for 100 sample stars (comprising normal stars as well as $\mathrm{Am} / \mathrm{HgMn}$ stars), motivated by the situation that systematic investigation on these important light elements had been lacking, and arrived at the following results:

- (1) Almost all stars (irrespective of being classified as peculiar or normal, though with a tendency of larger anomaly for the former) show more or less CNO deficits typically in

observations revealed that weak magnetic fields do exist even in Am or HgMn stars. 
the range of $-1 \lesssim[\mathrm{C}, \mathrm{N}, \mathrm{O} / \mathrm{H}] \lesssim 0$; i.e., no stars turned out to show normal CNO abundances. Besides, distinctly large deficiencies as much as $\sim 2$ dex are also shown for $\mathrm{C}$ or $\mathrm{N}$ by some CP stars $([\mathrm{C} / \mathrm{H}]$ for late Am stars or $[\mathrm{N} / \mathrm{H}]$ for $\mathrm{HgMn}$ stars of late B-type).

- (2) More useful information could be extracted by concentrating on the homogeneous sample of 16 A-type stars belonging to the Hyades cluster, which must have the same age and the same initial composition. In this way, we could confirm the systematic increase of abundance peculiarity with a decrease in $v_{\mathrm{e}} \sin i$ (projected rotational velocity), which manifestly indicates that rotation is the key factor responsible for controlling the extent of Am anomaly.

Therefore, result (1) suggests that CNO abundances are useful indicators for judging the degree of chemical peculiarity, which are applicable to wide variety of late B through early $\mathrm{F}$ stars regardless of whether or not being classified as peculiar. Besides, we learn from result (2) that studying and comparing the abundances of stars sharing homogeneous character (e.g., born at the same time with the same composition) is quite an effective approach for clarifying the nature of chemical anomaly.

It thus occurred to us to pay attention to the behavior of $\mathrm{CNO}$ abundances in the components of double-line spectroscopic binaries, which must have formed concurrently from the same gas such as the case of cluster stars. Distinct merits are also accompanied especially with eclipsing close binary systems, because stellar parameters (mass $M$, radius $R$, orbital inclination $i_{\text {orb }}$, etc) are well defined. In addition, since similar rotational velocities are realized for both components due to tidal synchronization, we would be able to check whether any other factor (apart from stellar rotation) affecting the chemical anomaly exists. Unfortunately, it is not an easy task to establish the abundances for both components of a spectroscopic binary, because lines of two stars are overlapped in a complex time-variable way depending on the orbital phase. Actually, such abundance studies of direct analyzing double-line spectra, which have occasionally been carried out since old days, are not regarded as sufficiently reliable, especially when weak lines are to be measured in a crowded spectrum.

However, the situation has dramatically changed nowadays thanks to progress of spectrum disentangling technique (e.g., Ilijić 2004 and the references therein), by which the genuine spectra of primary/secondary can be separately extracted from a set of double-line spectra sufficiently covering various orbital phases, making precise abundance determinations feasible for both components. Nevertheless, past applications of this technique to studying compositional differences of spectroscopic binaries comprising CP stars of late B through early F-type under question (Am stars, HgMn stars) are still only a few in number to our knowledge; e.g., Folsom et al. (2010) for AR Aur (B9V+B9.6V), Pavlovski et al. (2014) for YZ Cas (A2IV+F2V), and Torres et al. (2015)

2 Some authors prefer to use the term "disentangling" to specifically imply iterative determination of orbital elements, which requires reliable radial velocities based on appropriately isolated spectra of both components, while simply using "separation" if only spectrum reconstruction of each component is involved. Note, however, that we use "disentangling" in the latter meaning in this paper. for V501 Mon (A6m+F0); and besides these studies do not seem to have paid much attention to $\mathrm{CNO}$ abundances of our interest.

Motivated by this situation, we decided to investigate the abundances of CNO (as well as several other elements derived as by-products) and how they differ between the components for 5 eclipsing binary systems comprising CP stars (AR Aur, $\beta$ Aur, YZ Cas, WW Aur, and RR Lyn), based on the adequately disentangled spectra of the primary and secondary, in essentially the same manner as done in Paper I. These 5 systems, which were selected because sufficient observational data covering various phases (necessary for spectrum disentangling) are available, can be divided into two groups: "similar-component" group (AR Aur with late-B stars, $\beta$ Aur with early-A stars, and WW Aur with mid-A stars) and "different-component" group (YZ Cas with early-A and early-F stars, RR Lyn with late-A and early-F stars). In what follows are the check points we would like to elucidate in this study.

- All the component stars of our targets (with spectral types of late B through early $\mathrm{F})$ are slow rotators $\left(v_{\mathrm{e}} \sim 10\right.$ $40 \mathrm{~km} \mathrm{~s}^{-1}$ ) because rotation and orbital motion are almost synchronized in such close binary systems (orbital period is $P \sim 3-10$ day). Then, according to our knowledge, we may expect that all these stars would more or less exhibit chemical peculiarities. Could we really confirm this?

- Regarding the systems of "similar-component" group, almost the same abundances should be observed for both components according to our naive expectation because differences in stelar parameters (including rotational velocities) are fairly small. What about the observational results on this point?

- As to the binary systems belonging to "differentcomponent" group, the primary and secondary stars have distinctly different $T_{\text {eff }}$ from each other. Could we detect any compositional differences between the two? If so, this may provide us with useful information regarding $T_{\text {eff- }}$ dependence of chemical peculiarities.

\section{OBSERVATIONAL DATA}

\subsection{Observations}

The observations of our 5 program stars (AR Aur, $\beta$ Aur, YZ Cas, WW Aur, and RR Lyn; cf. Table 1 for the basic data) were carried out on 2010 December 14, 15, 16, 18, and 20 by using BOES (Bohyunsan Observatory Echelle Spectrograph) attached to the $1.8 \mathrm{~m}$ reflector at Bohyunsan Optical Astronomy Observatory. Using $2 \mathrm{k} \times 4 \mathrm{k}$ CCD (pixel size of $15 \mu \mathrm{m} \times 15 \mu \mathrm{m}$ ), this echelle spectrograph enabled us to obtain spectra of wide wavelength coverage (from $\sim 3800 \AA$ to $\sim 9200 \AA$ ). We used $200 \mu \mathrm{m}$ fiber corresponding to the resolving power of $R \simeq 45000$. The total integration time for one observation (consisting of $2-3$ successive frames to be co-added) was chosen to be $\sim 10-60$ minutes depending on the brightness of a target. Each of the program stars were observed 1-3 times in a night with an interval of a few hours, though the actual frequency differed from star to star. Thus, as a result of 5-night observations, we could obtain 53 spectra for these 5 targets, which consist of 9-11 spectra per each star corresponding to different observational times. The 
fundamental information (spectrum code and observational time in Julian day) for each of the 53 spectra is presented in Table 2.

The reduction of the echelle spectra (bias subtraction, flat fielding, spectrum extraction, wavelength calibration, and continuum normalization) was carried out by using the software package IRAF (Image Reduction and Analysis Facility) 3

\subsection{Spectrum disentangling}

It is essential to correctly specify the local radial velocities for the primary $\left(V_{1}^{\text {local }}\right)$ and the secondary $\left(V_{2}^{\text {local }}\right)$ corresponding to each spectrum, in order to successfully obtain the disentangled spectra of both components. For this purpose, we first evaluated the predicted $V_{1}^{\text {local }}$ and $V_{2}^{\text {local }}$ at each relevant observational time as presented in Table 2 (7th and 8th columns), where we employed the ephemeris data of primary minimum (taken from the web site of General Catalogue of Variable Stars; Samus et al. 2017) and the orbital elements (taken from the compilation of Eker et al. 2014) summarized in Table 1 . Then, we simulated the theoretical double-line profile of O I 7771-5 triplet corresponding to such evaluated $V_{1}^{\text {local }}$ and $V_{2}^{\text {local }}$ by using the spectrumsynthesis technique, which was further compared with the observed profile in order to check whether both agree well with each other. While a satisfactory consistency was confirmed for $\beta$ Aur, YZ Cas, and WW Aur, small systematic differences (due to slight variation of system velocity?) were seen for AR Aur and appreciable phase-dependent disagreements (likely to be caused by inadequately computed phases) were observed for RR Lyn 5 Therefore, we estimated the empirical radial velocities $\left(V_{1}^{\mathrm{emp}}\right.$ and $\left.V_{2}^{\mathrm{emp}}\right)$ by directly comparing the theoretical O I 7771-5 line profiles (simulated for various combinations of $V_{1}$ and $V_{2}$ ) with the observed one. These various kinds of radial velocities evaluated for each of the spectra are summarized in Table 2 . The radial velocities of both components finally adopted for disentangling $\left(V_{1}^{\text {local }}\right.$ and $V_{2}^{\text {local }}$ for $\beta$ Aur, YZ Cas, and WW Aur; $V_{1}^{\text {emp }}$ and $V_{2}^{\text {emp }}$ for AR Aur and RR Lyn) are graphically depicted by symbols (converted to the heliocentric scale) in Fig. 1, where the predicted radial velocity curves are also shown for comparison. Note that 2 spectra for WW Aur (20101216A) and RR Lyn (20101214A) corresponding to eclipse phases were eventually not used, as indicated in Fig. 1 (by arrows) as well as in Table 2 (by parentheses).

We selected four wavelength regions of 50-60 A width, for which disentangled spectra of both components are to be extracted: 5350-5400 $\AA, 6130-6180 \AA, 7440-7500 \AA$, and 7750-7800 $\AA$. The first three are obvious because we intend

3 IRAF is distributed by the National Optical Astronomy Observatories, which is operated by the Association of Universities for Research in Astronomy, Inc. under cooperative agreement with the National Science Foundation.

4 Available at http://www.sai.msu.su/gcvs/gcvs/index.htm

5 Although we refrain from discussing the reason for the discrepancy, possible anomalies of radial velocity curves have occasionally been mentioned for these two stars. See, e.g., Wilson \& Van Hamme (2014; Sect. 8 and the references therein) for AR Aur and Tomkin \& Fekel (2006; Sect. 6.1 and the references therein) for RR Lyn. to determine the abundances of $\mathrm{C}, \mathrm{N}$, and $\mathrm{O}$ based on $\mathrm{C}$ I 5380, N I 7468, and O I 6156-8 lines, closely following Paper I. The inclusion of $7750-7800 \AA$ region is to make use of the $\mathrm{O}$ I $7771-5$ triplet lines, which are so strong and saturated as to be applied to checking $v_{\mathrm{t}}$ (microturbulence) and to precisely determining $v_{\mathrm{e}} \sin i$ (cf. Sect. 4.1). Another parameter necessary to specify for spectrum disentangling is the luminosity ratio of the primary and secondary $\left(l_{1}: l_{2}\right)$ relevant for each wavelength region. We computed this ratio by using the emergent continuum fluxes $\left(F_{1}\right.$ and $\left.F_{2}\right)$ computed from Kurucz's (1993) ATLAS9 program (corresponding to $T_{\text {eff }}$ and $\log g$ for each star) and the stellar radii $\left(R_{1}\right.$ and $\left.R_{2}\right)$ as $l_{1}: l_{2}=F_{1} R_{1}^{2}: F_{2} R_{2}^{2}$, where the stellar parameters were taken from Table 1 . The resulting luminosity ratios are summarized in Table 3.

Regarding the software tool for spectrum disentangling, we made use of the public-domain program CRES6 written by S. Ilijić, as employed by Takeda, Hashimoto \& Honda (2018b) for their study of Capella's photospheric abundances. See Sect. 3 of their paper for more details of the procedures we adopted. We confirmed that the resulting disentangled spectra turned out quite satisfactory. The accomplished S/N ratios are sufficiently high $(\sim 300-700)$, except for RR Lyn (2) ( 100-200) and YZ Cas (2) ( 70-90), as summarized in Table 4. Fig. 2 demonstrates how the pure primary and secondary spectra could be successfully extracted from a set of original spectra in the neighborhood of $\mathrm{O}$ I 7771-5. All these disentangled spectra (for 10 stars and 4 regions) used in this study are presented as on-line material (file name: all_spectra).

Finally, it should be remarked that our application of the spectrum disentangling method implicitly assumed that the line spectra and the brightness of each component did not show any time variability during the observed period, despite that the existence of small intrinsic spectrum variability (caused by chemical inhomogeneity on the surface?) has been reported for some of our program stars; e.g., for WW Aur (cf. Kitamura, Kim \& Kiyokawa 1976) and AR Aur (cf. Folsom et al. 2010 and the references therein).

\section{ABUNDANCE DETERMINATION}

\subsection{Atmospheric parameters}

Regarding $T_{\text {eff }}$ and $\log g$ necessary to specify the model atmosphere for each star, we adopted the values compiled by Eker et al. (2014) in this study (cf. Table 1). Here, we should note that, while these $\log g$ values are regarded as fairly precise (quoted errors are only 0.01-0.03 dex) for these eclipsing binary stars because $M$ and $R$ are well established, $T_{\text {eff }}$ 's are the photometry-based values and may not be so accurate. In Fig. 3 are plotted our 10 program stars on the $T_{\text {eff }}$ vs. $\log g$ plane, where theoretical evolutionary tracks of solarmetallicity stars computed for various $M$ values are also depicted. We can see from this figure that the track-based $M$ is not consistent with the real $M$ for some cases (e.g., for WW Aur or RR Lyn), for which $T_{\text {eff }}$ errors even larger than the nominal values $(\sim 100-400 \mathrm{~K})$ may be possible. The microturbulent velocities $\left(v_{\mathrm{t}}\right)$ were derived from $T_{\text {eff }}$ by

${ }^{6}$ http://sail.zpf.fer.hr/cres/ 
using the empirical relation [Eq. (1) in Takeda et al. (2008)] as done in Paper I, though appreciable ambiguities may be involved with them (cf. Sect. 4.1). In estimating abundance errors due to uncertainties in atmospheric parameters, we tentatively assume in this study $\pm 500 \mathrm{~K}$ in $T_{\text {eff }}, \pm 0.1 \mathrm{dex}$ in $\log g$, and $\pm 50 \%$ in $v_{\mathrm{t}}$. for all stars (which may be regarded rather as upper limits of actual errors).

\subsection{Procedures}

The determination of CNO abundances (synthetic spectrum fitting, evaluation of equivalent widths, error estimation) was done in essentially the same manner as adopted in $\mathrm{Pa}$ per I, which should be consulted for the details (cf. Sect. 4 therein). We here remark only some differences specific to this study.

- The O I 7771-5 triplet lines are also analyzed based on the $7750-7800 \AA$ fitting, in a similar manner to the case of O I $6156-8$, though this is mainly for the derivation of $v_{\mathrm{e}} \sin i$ as well as for checking $v_{\mathrm{t}}$ (rather than for O-abundance determination).

- The wavelength range of spectrum fitting targeting $\mathrm{O}$ I 6156-8 lines was extended somewhat shortwards to include Ba II 6141 line, in order to get information of Ba abundance.

- We make use of the abundances of various elements other than CNO (specifically, $\mathrm{Na}, \mathrm{Si}, \mathrm{Ca}, \mathrm{Ti}, \mathrm{Fe}$, and $\mathrm{Ba}$ ), which are derived as by-products of spectrum fitting (though under the assumption of LTE), since they also serve as useful indicators of chemical peculiarities.

The details of spectrum fitting analysis (wavelength range, source of line data, varied abundances) done for four regions are presented in Table 5 , and the basic atomic data of $\mathrm{C}$ I $5380, \mathrm{~N}$ I $7468, \mathrm{O}$ I $6156-8$, and $\mathrm{O}$ I $7771-5$ lines are summarized in Table 6 . The accomplished consistency between the theoretical spectrum (for the converged solutions) and the observed spectrum as a result of our fitting analysis is displayed in Fig. $4-7$ for each region. In Fig. 8 are plotted the resulting equivalent width $(W)$, non-LTE correction $(\Delta)$, and non-LTE abundance $\left(A^{\mathrm{N}}\right)$ for each of these CNO lines against $T_{\text {eff }}$, just like Fig. $5-7$ of Paper I. More complete results of these $\mathrm{CNO}$ abundances (including errors and related quantities) and the abundances of other elements ( $\mathrm{Na}, \mathrm{Si}, \mathrm{Ca}, \mathrm{Ti}, \mathrm{Fe}$, and $\mathrm{Ba}$ ) resulting from spectrum fitting as by-products are presented as online material (filename: abundances.dat). Besides, the abundances of $\mathrm{Na}$, $\mathrm{Si}, \mathrm{Ca}$, and Ti for 100 A-type main-sequence stars targeted in Paper I, which were not published therein because the main focus was placed only on $\mathrm{CNO}$, are also given as online material (filename: paperI_suppl.dat) for reference.

\subsection{Impact of errors in the luminosity ratio}

Here, as an additional error source specific to this study, it may be worth mentioning the effect of uncertainties in the luminosity ratio adopted for spectrum disentangling (cf. Sect. 2.2), since the emergent flux $(F)$ would suffer some ambiguities due to errors in $T_{\text {eff }}$ (typically by a few hundred K; cf. Sect. 3.1), although the radii $(R)$ of both stars are precisely determined. Since $F$ is changed by $\sim 20-30 \%$ for an increase in $T_{\text {eff }}$ by $1000 \mathrm{~K}$, errors of several to $\lesssim 10 \%$ may be possible in the computed luminosity. As a test trial, we carried out spectrum disentangling in the $6130-6180 \AA$ region by increasing the $l_{1} / l_{2}$ ratio by $10 \%$ (e.g., $0.542: 0.458$ instead of 0.518:0.482 for AR Aur or 0.793:0.207 instead of 0.777:0.223 for RR Lyn), and determined the abundances of $\mathrm{O}, \mathrm{Na}, \mathrm{Si}, \mathrm{Ca}, \mathrm{Fe}$, and $\mathrm{Ba}$ in the same manner based on such specially reconstructed spectra with intentionally increased $l_{1} / l_{2}$. Comparing the resulting abundances with the standard values, we found that the differences are generally insignificant (typically several hundredths dex), except for the strong/deep line cases of $\mathrm{Ba}$ or $\mathrm{Ca}$ (cf. Fig 6) where more appreciable abundance changes of $\sim 0.1-0.15$ dex are seen (especially for YZ Cas (2) and RR Lyn (2)). This may be interpreted as due to the fact that an error in the zero-point level of the residual intensity, which is caused by inappropriately chosen luminosity ratio, significantly affects deep/strong lines but not shallow/weak lines (see footnote 12 in Takeda, Hashimoto, \& Honda 2018b). Accordingly, as far as the abundances of $\mathrm{C}, \mathrm{N}$, and $\mathrm{O}$ derived from C I 5380, N i 7468, and O I 6156-8 lines (our main objective which we will discuss in Sect. 4.2) are concerned, this kind of error is of negligible importance, because these lines are sufficiently weak.

\section{DISCUSSION}

\subsection{Microturbulence and rotation}

We start by mentioning about the stellar parameters $\left(v_{\mathrm{t}}\right.$ and $v_{\text {e }} \sin i$ ) related to $\mathrm{O}$ I $7771-5$ triplet, which was specially analyzed in this study.

Regarding the microturbulence, we simply applied the empirical $T_{\text {eff-dependent analytical formula proposed by }}$ Takeda et al. (2008), as done in Paper I. A useful test for examining the adequacy of such assumed $v_{\mathrm{t}}$ is to check whether the non-LTE oxygen abundances derived from strong $\mathrm{O}$ I $7771-5$ lines $\left(A_{77}^{\mathrm{N}}\right)$ and weak O I $6156-8$ lines $\left(A_{61}^{\mathrm{N}}\right)$ agree with each other, because the former is appreciably $v_{\mathrm{t}}$ sensitive while the latter is not. Such a comparison is displayed in Fig. 9a, indicating that $A_{77}^{\mathrm{N}}$ tends to be somewhat larger than $A_{61}^{\mathrm{N}}$ (this difference is also recognized by comparing Fig. 8c and Fig. 8d). We can determine the "abundance-matched" microturbulence $v_{\mathrm{t}}(\mathrm{A})$ by requiring the abundance equality $\left(A_{77}^{\mathrm{N}}=A_{61}^{\mathrm{N}}\right.$; cf. Fig $\left.9 \mathrm{~b}\right)$. These $v_{\mathrm{t}}(\mathrm{A})$ values are plotted against $T_{\text {eff }}$ in Fig. $9 \mathrm{c}$, which shows that these values are somewhat larger than the analytical relation we adopted (as expected). For reference, we also derived the "profile-based" microturbulence $v_{\mathrm{t}}(\mathrm{P})$, which was so determined as to accomplish the best-fit $\mathrm{O}$ I $7771-5$ triplet line profile (cf. Sect. 5 in Takeda, Jeong \& Han 2018a). Such obtained $v_{\mathrm{t}}(\mathrm{P})$ results are depicted in Fig. 9d, where we see that $v_{\mathrm{t}}(\mathrm{P})$ tends to become higher than the adopted relation at $T_{\text {eff }} \lesssim 8000 \mathrm{~K}$. Taking all these results into consideration, we conservatively assumed that uncertainties of $\pm 50 \%$ may be involved with the $v_{\mathrm{t}}$ values adopted in this study, as mentioned in Sect. 3.1. Fortunately, the CNO abundances derived from C I 5380, N I 7468, and O I 6156-8 lines (which are all quite weak) are hardly affected by any choice of $v_{\mathrm{t}}$, though abundances from stronger saturated lines (e.g., Ba abundance from $\mathrm{Ba}$ II 6141 line) are considerably $v_{\mathrm{t}}$ dependent. 
Since the O I 7771-5 triplet is almost free from blending with other lines and shows a characteristic profile comprising three component lines (which sometimes show a complex substructure by merging of rotationally broadened profiles; see, e.g., Fig. 7b or Fig. 7d), precise determination of $v_{\mathrm{e}} \sin i$ may be expected if successful fitting between theoretical and observed profiles could be accomplished. Such spectroscopically established $v_{\mathrm{e}} \sin i$ values (presented in Table 1) can be used to check whether the expected synchronization of rotational and orbital motions are actually realized in these close binary systems. Assuming the alignment of orbital and rotational axes, we can derive the observed equatorial rotation velocity $\left(v_{\mathrm{e}}\right)$ by dividing $v_{\mathrm{e}} \sin i$ by the sinus of orbital inclination $\left(i_{\mathrm{orb}}\right)$. The comparison of such obtained $v_{\mathrm{e}}$ with $V_{\text {syn }}$ ( $\equiv 2 \pi R / P$ : synchronized rotation velocity) is shown in Fig. 10a, where can see that the agreement is mostly satisfactory. This suggests that synchronization is almost realized in these close binary systems, which lends support to the result of Kitamura \& Kondo (1978) who arrived at practically the same conclusion. However, the situation is indefinite regarding the comparatively longer period case of RR Lyn ( $P \simeq 10$ day), where $v_{\mathrm{e}}$ is apparently larger than $V_{\text {syn }}$ by $\sim 20-40 \%$ (cf. Fig. 10b). This might indicate that co-rotation has not yet been realized in this system. Alternatively, we can not rule out a possibility of overestimated $v_{\mathrm{e}} \sin i$ for such a sharp-lined star $\left(\sim 10 \mathrm{~km} \mathrm{~s}^{-1}\right)$ due to our neglect of some significant broadening component (e.g., macroturbulence), although the effect of instrumental broadening (corresponding to the spectrum resolving power of $R \simeq 45000$ ) was taken into consideration in deriving $v_{\mathrm{e}} \sin i$.

\subsection{Abundance characteristics}

We now discuss the abundances of 10 stars (primary and secondary of 5 systems) resulting from our analysis. Regarding oxygen, we exclusively use the abundance derived from O I 6156-8 lines (as done in Paper I), because the abundance from $\mathrm{O}$ I $7771-5$ is sensitive to $v_{\mathrm{t}}$ and suffers a large non-LTE effect. The differential abundances relative to the standard star Procyon 7 ( $[\mathrm{X} / \mathrm{H}] ; \mathrm{X}$ is any of $\mathrm{C}, \mathrm{N}, \mathrm{O}, \mathrm{Na}$, $\mathrm{Si}, \mathrm{Ca}, \mathrm{Ti}, \mathrm{Fe}$, and $\mathrm{Ba}$ ) for each star are summarized in $\mathrm{Ta}-$ ble 7 and graphically plotted in Fig. 11. We can read from this figure several characteristic trends in terms of the check points set up in Sect. 1 .

- Regarding the systems belonging to "similar component" group (AR Aur, $\beta$ Aur, WW Aur), the abundances of the primary and secondary components are very similar and hardly discernible for $\beta$ Aur and WW Aur, while those for AR Aur are apparent different. More precisely, both components of $\beta$ Aur and WW Aur show the characteristics of Am phenomena: $\mathrm{C} / \mathrm{N} / \mathrm{O}$ are deficient by several tenths to $\lesssim 1$ dex (cf. Paper I), Si/Na/Ti/Fe are normal/enriched, $\mathrm{Ca}$ is normal/underabundant, and $\mathrm{Ba}$ is conspicuously overabundant (see, e.g., Fig. 9 in Varenne \& Monier 1999). In contrast, the primary component of AR Aur shows a clear characteristics of HgMn stars (this star is actually known to

7 The surface composition of this star is known to be almost the same as that of the Sun. See the references quoted in Sect. IV(c) of Takeda et al. (2008). be of this type) in the sense that $\mathrm{N}$ is drastically depleted by $\gtrsim 2$ dex, whereas the secondary appears to exhibit some weak Am phenomenon as seen from moderate deficiencies of $\mathrm{CNO}$ and large overabundance of Ba.

- As for YZ Cas and RR Lyn belonging to "differentcomponent" systems, the surface composition of the hotter primary is appreciably different from that of the cooler secondary. That is, the former shows clear Am-type peculiarities mentioned above, while the latter is nearly normal within a few tenths dex without showing any conspicuous anomaly despite of slow rotators. It is worth noting that these normal-composition secondary stars of YZ Cas and RR Lyn are at $T_{\text {eff }} \lesssim 7000 \mathrm{~K}$.

Combining these observational facts, we can draw the following consequences regarding the appearance and properties of chemical peculiarities in late $\mathrm{B}$ through early $\mathrm{F}$ stars.

- The fact that essentially the same Am peculiarities are observed between the similar primary and secondary (quite similar $v_{\mathrm{e}} \sin i$ and only small $T_{\text {eff }}$ difference of $\left.\sim 200-300 \mathrm{~K}\right)$ for both $\beta$ Aur and WW Aur means that Am phenomenon is mainly determined by $v_{\mathrm{e}} \sin i$ and not very sensitive to $T_{\text {eff }}$ as far as the $T_{\text {eff }}$ range of $10000 \mathrm{~K} \gtrsim T_{\text {eff }} \gtrsim 7000 \mathrm{~K}$ is concerned. This is a reconfirmation of the primary importance of rotational velocity in the appearance of Am anomalies, though their extents show moderate $T_{\text {eff-dependences (e.g., }}$ tendency of larger CNO deficiency towards lower $\left.T_{\text {eff }}\right)$.

- In the context of essentially same chemical compositions between the primary and secondary for $\beta$ Aur and WW Aur, it is interesting to note that these two systems show markedly different $\mathrm{Na}$ as well as $\mathrm{Ca}$ abundances from each other (cf. Fig. 11b,d). We consider that this discrepancy is mainly due to the difference in $T_{\text {eff }}$ (by $\sim 1500 \mathrm{~K}$ ), since $[\mathrm{Na} / \mathrm{H}]$ and $[\mathrm{Ca} / \mathrm{H}]$ tend to decrease with a lowering of $T_{\text {eff }}$ as can be recognized in Fig. 12 , where the $[\mathrm{Na} / \mathrm{H}]$ and $[\mathrm{Ca} / \mathrm{H}]$ values of 100 A-type stars studied in Paper I are plotted against $T_{\text {eff }}$. Likewise, diffusion calculation predicts the similar tendency at least for Ca (see, e.g., Fig. 14 in Richer, Michaud \& Turcotte 2000). Although these two stars have distinctly different ages, $\sim 450-500 \mathrm{Myr}$ for $\beta$ Aur (Southworth, Bruntt \& Buzasi 2007) and 90 Myr for WW Aur (Southworth et al. 2005), it seems rather unlikely that this has caused the abundance difference, because $\mathrm{Ca}$ is considerably deficient in younger WW Aur while nearly normal in older $\beta$ Aur (i.e., in conflict with the naive expectation that chemical anomaly would develop with time).

- The apparent abundance difference between AR Aur (1) (HgMn star) and AR Aur (2) (weak Am star) despite their similarity in stellar parameters (essentially the same rotational velocity and only a small $T_{\text {eff }}$ difference of $600 \mathrm{~K}$ ) is somewhat strange. It may be possible that a rather distinct transition occurs somewhere in-between $T_{\text {eff }} \sim 10000$ $11000 \mathrm{~K}$ regarding the chemical peculiarity of slow rotators; i.e., from Am-type to HgMn-type as $T_{\text {eff }}$ is increased.

- The chemical composition difference between the primary (Am) and the secondary (normal) seen in YZ Cas $\left(T_{\text {eff }, 1} / T_{\text {eff }, 2}=9200 \mathrm{~K} / 6700 \mathrm{~K}\right)$ and RR Lyn $(7570 \mathrm{~K} / 6980 \mathrm{~K})$ provides us with important information concerning the $T_{\text {eff-dependence for the appearance of } \mathrm{Am}}$ stars. Especially, the distinct transition from Am to normal over a small $T_{\text {eff }}$ difference of $\sim 600 \mathrm{~K}$ for the case of RR Lyn 
is significant. We can state from these facts that the advent of Am phenomenon is confined to $T_{\text {eff }} \gtrsim 7000 \mathrm{~K}$, below which chemical peculiarities do not appear no matter how slowly a star rotates. Actually, we can see from Fig. $8 \mathrm{e}-8 \mathrm{~g}$ of Paper I that the large spread of $\mathrm{C}, \mathrm{N}$, and $\mathrm{O}$ abundance deficiencies tend to quickly shrink at $T_{\text {eff }} \sim 7000 \mathrm{~K}$. This is presumably related to the thickening of surface convection zone counteracting the formation of chemical anomaly.

Further, in order to visualize these abundance characteristics summarized above, we plot in Fig. 13 the values of $[\mathrm{C} / \mathrm{H}],[\mathrm{N} / \mathrm{H}]$, and $[\mathrm{O} / \mathrm{H}]$ for the 10 program stars as well as those of 100 stars (Hyades stars and field stars) taken from Paper I altogether against $T_{\text {eff }}$ and $v_{\mathrm{e}} \sin i$. It can be recognized from this figure that the $\mathrm{CNO}$ abundances of the close binary stars are more or less consistent with the results for general A-type stars. Especially, our results (except for YZ Cas (2) and RR Lyn (2), which are normal abundance stars at $T_{\text {eff }} \lesssim 7000 \mathrm{~K}$ ) reasonably follow the systematic $[\mathrm{X} / \mathrm{H}]$ vs. $v_{\mathrm{e}} \sin i$ trend established by Hyades stars (small open circles) despite the narrow range of $v_{\mathrm{e}} \sin i$ $\left(\sim 10-40 \mathrm{~km} \mathrm{~s}^{-1}\right)$, which reaffirms the importance of rotational velocity for the build-up of Am peculiarity.

\subsection{Comparison with previous studies}

Finally, we briefly comment on how the results derived from our spectroscopic analysis of 5 binary systems are compared with similar chemical abundance studies published so far. Here, focus of attention is placed on whether or not these previous investigations are consistent with our consequences.

\subsubsection{AR Aur}

Khokhlova et al. (1995) concluded in their chemical abundance study of AR Aur for 18 elements (based on the line strengths of each component carefully measured from the double-lined spectra of various phases) that the primary is a typical HgMn star while the secondary is also abnormal but with different type of peculiarity. Similarly, according to Folsom et al.'s (2010) more recent investigation based on the disentangled spectra of AR Aur, the surface chemical compositions of the primary and secondary are apparently different, indicating that the former is a $\mathrm{HgMn}$ star and the latter is a weak Am star (cf. Fig. 4 therein). Therefore, our conclusion is almost consistent with these past studies. Although Folsom et al. (2010) reported that the C and O abundances are nearly solar for both components (in contrast to our result of mild but definite deficiency), this may be partly due to their neglect of the non-LTE effect.

\subsection{2 $\beta$ Aur}

Lyubimkov, Rachkovskaya \& Rostopchin (1996) determined the chemical compositions of the primary and secondary stars of $\beta$ Aur by analyzing the double-lined spectra, and concluded that the abundances of both components are quite similar and show the characteristics of Am stars. The abundance characteristics of various elements (including $\mathrm{CNO}$ ) reported by them are in satisfactory agreement with our results.

\subsubsection{YZ Cas}

Pavlovski et al. (2014) recently carrird out chemical abundance determinations separately for each of the components of YZ Cas based on the disentangled spectra. They reported that the primary is an Am star with supersolar abundances (except for Sc) while the composition of secondary is nearly solar, which is almost consistent with our conclusion. However, their results of "supersolar" C and O abundances (like other heavier elements) for the Am primary are in serious conflict with the general trend of CNO deficiency confirmed in Paper I as well as in this study. Unfortunately, since they did not publish any details of their analysis (e.g., used lines, equivalent widths), we have no idea about the reason for this discrepancy.

\subsubsection{WW Aur}

To our knowledge, no chemical abundance study for this binary system has ever been published so far. Although Pavlovski, Southworth \& Tamajo (2008) once reported their accomplishment of spectrum disentangling applied to WW Aur, stating that the related abundance analysis was in progress, their successive paper does not seem to have come out yet.

\subsubsection{RR Lyn}

Several spectroscopic investigations have already been published since old days regarding the chemical abundances of RR Lyn (Popper 1971; Kondo 1976; Burkhart \& Coupry 1991; Lyubimkov \& Rachkovskaya 1995; Hui-BonHoa 2000), although all these are based on the direct analysis of double-lined spectra. According to these previous studies, the primary component is an Am star and more metal-rich than the secondary, which is in reasonable consistency with our consequence. However, none of them mentioned about the CNO abundances of this binary system.

\section{CONCLUSION}

Spectroscopically investigating the chemical compositions separately for the primary and secondary components of a double-line eclipsing binary showing characteristic chemical peculiarities (typically seen in A-tye stars in the upper main sequence) is of profound significance, since it may provide us with information regarding how the appearance of chemical anomaly depends upon stellar physical parameters.

We conducted a spectroscopic study to determine the surface chemical abundances of CNO and other heavier elements in the primary and secondary components of selected 5 eclipsing close binaries around A-type (AR Aur, $\beta$ Aur, YZ Cas, WW Aur, and RR Lyn). Our aim was to investigate whether any chemical difference exists between two components, and how the situation depends upon stellar parameters.

For this purpose, we extracted the individual spectra of the primary and secondary for each target in 4 wavelength regions by applying the spectrum disentangling method to a set of time-series spectra sufficiently covering the orbital phases, which were obtained by using the high-dispersion 
echelle spectrograph attached to the $1.8 \mathrm{~m}$ reflector of Bohyunsan Optical Astronomy Observatory.

The abundances of $\mathrm{C}, \mathrm{N}$, and $\mathrm{O}$ were determined by using C I 5380, N I 7468, and O I 6156-8 lines in essentially the same manner (spectrum-synthesis analysis by taking into account the non-LTE effect) as done in Paper I. Besides, we could also derive the abundances of $\mathrm{Na}, \mathrm{Si}, \mathrm{Ca}, \mathrm{Ti}, \mathrm{Fe}$, and $\mathrm{Ba}$ as by-products of spectrum fitting.

In addition, we also analyzed the $\mathrm{O}$ I $7771-5$ triplet lines in the same way, with an aim to check the adopted $v_{\mathrm{t}}$ and to determine $v_{\mathrm{e}} \sin i$ for each star. We could confirm that synchronization between rotation and orbital motion is accomplished for almost all systems, except for RR Lyn for which the situation is unclear.

Regarding the "similar-component" systems, $\beta$ Aur and WW Aur were confirmed to exhibit the characteristics of Am anomaly (deficiency in CNO, overabundance of heavy elements such as $\mathrm{Ba}$ ) with essentially the same compositions for both the primary and secondary.

However, distinct compositional differences were found between the primary and secondary of AR Aur, another "similar-component" system, in the sense that they show chemical anomalies of different types (HgMn star and Am star, respectively).

In contrast, as to YZ Cas and RR Lyn, both of which are the "different-component" systems consisting of A-type and early-F stars, the surface abundances turned out markedly different between the primary (Am star) and secondary (chemically normal).

These observational results may indicate the $T_{\text {eff- }}$ dependent nature in the chemical anomalies of non-magnetic stars on the upper main sequence, as summarized below.

-(i) In the effective temperature range of $10000 \mathrm{~K} \gtrsim T_{\text {eff }} \gtrsim$ $7000 \mathrm{~K}$, slow rotation is considered to be the key factor for the appearance of Am anomaly. Thus, the components of close binaries (slow rotators due to tidal braking) naturally exhibit very similar Am anomalies under the same rotational velocities ( $\beta$ Aur and WW Aur).

-(ii) However, the emergence of Am peculiarity seems to have a distinct lower $T_{\text {eff }}$ limit at $\sim 7000 \mathrm{~K}$, below which abundance anomaly can hardly be produced regardless of stellar rotation (secondary components of RR Lyn and YZ Cas).

-(iii) On the other hand, as seen from the abundance differences between the components of AR Aur, the transition from Am anomaly to $\mathrm{HgMn}$ anomaly is likely to take place (with an increase in $T_{\text {eff }}$ ) somewhere between $\sim 10000 \mathrm{~K}$ and $\sim 11000 \mathrm{~K}$.

\section{ACKNOWLEDGMENTS}

We express our cordial thanks to Dr. S. Ilijić for kindly making the useful spectrum disentangling software CRES open to the public. Data reduction and analysis were in part carried out by using the common-use data analysis computer system at the Astronomy Data Center (ADC) of the National Astronomical Observatory of Japan.

\section{REFERENCES}

Bressan A., Marigo P., Girardi L., Salasnich B., Dal Cero C., Rubele S., Nanni A., 2012, MNRAS, 427, 127

Burkhart C., \& Coupry M. F., 1991, A\&A, 249, 205

Eker Z., Bilir S., Soydugan F., Yaz Gökçe E., Soydugan E., Tüysüz M., Şenyüz T., Demircan O., 2014, PASA, 31, e024

Folsom C. P., Kochukhov O., Wade G. A., Silvester J., Bagnulo S., 2010, MNRAS, 407, 2383

Hui-Bon-Hoa A., 2000, A\&AS, 144, 203

Ilijić S. 2004, in Spectroscopically and Spatially Resolving the Components of Close Binary Stars, ASP Conf. Ser. Vol. 318, ed. R. W. Hilditch, H. Hensberge, \& K. Pavlovski (San Francisco: Astronomical Society of the Pacific), 107

Khokhlova V. L., Zverko Yu.,Zhizhnovskii I., Griffin R. E. M., 1995, Astron. Lett., 21, 818

Kitamura M., Kim T.-H., Kiyokawa M., 1976, Ann. Tokyo Astron. Obs. 2nd. Ser., 16, 22

Kitamura M., Kondo M., 1978, Ap\&SS, 56, 341

Kondo M., 1976, Ann. Tokyo Astron. Obs. 2nd. Ser., 16, 1

Kurucz R. L., 1993, Kurucz CD-ROM, No. 13, ATLAS9 Stellar Atmosphere Program and $2 \mathrm{~km} / \mathrm{s}$ Grid (Cambridge, MA: Harvard-Smithsonian Center for Astrophysics)

Kurucz R. L., Bell B., 1995, Kurucz CD-ROM, No. 23, Atomic Line Data (Cambridge, MA: Harvard-Smithsonian Center for Astrophysics)

Lyubimkov L. S., Rachkovskaya, T. M., 1995 Astron. Rep., 39, 63

Lyubimkov L. S., Rachkovskaya T. M., Rostopchin S. I., 1996, Astron. Rep., 40, 802

Pavlovski K., Southworth J., Kolbas V., Smalley B., 2014, MNRAS, 438, 590

Pavlovski K., Southworth J., Tamajo E., 2008, Contrib. Astron. Obs. Skalnaté Pleso, 38, 437

Popper D. M., 1971, ApJ, 169, 549

Preston G. W., 1974, ARA\&A, 12, 257

Richer J., Michaud G., Turcotte S., 2000, ApJ, 529, 338

Samus N. N., Kazarovets E. V., Durlevich O. V., Kireeva N. N., Pastukhova E. N., 2017, Astron. Rep., 61, 80

Southworth J., Smalley B., Maxted P. F. L., Claret A., Etzel P. B., 2005, MNRAS, 363, 529

Southworth J., Bruntt H., Buzasi D. L., 2007, A\&A, 467, 1215

Takeda Y., Han I., Kang D.-I., Lee B.-C., Kim K.-M., 2008, JKAS, 41,83

Takeda Y., Hashimoto O., Honda S., 2018b, ApJ, 862, 57

Takeda Y., Jeong G., Han I., 2018a, PASJ, 70, 8

Takeda Y., Kawanomoto S., Ohishi N., Kang D.-I., Lee B.-C., Kim K.-M., Han I., 2018c, PASJ, 70, 91 (Paper I)

Takeda Y., Sadakane K., 1997, PASJ, 49, 571

Tomkin J., Fekel F. C., 2006, AJ, 131, 2652

Torres G., Lacy C. H. S., Pavlovski K., Fekel F. C., Muterspaugh M. W., 2015, ApJ, 150, 154

Varenne O., Monier R., 1999, A\&A, 351, 247

Wilson R. E., Van Hamme W., 2014, ApJ, 780, 151 
Table 1. Fundamental data of the program stars.

\begin{tabular}{|c|c|c|c|c|c|c|c|}
\hline & & AR Aur & $\beta$ Aur & YZ Cas & WW Aur & RR Lyn & Remark \\
\hline \multicolumn{8}{|c|}{ [Basic data] } \\
\hline HD & & 34364 & 40183 & 4161 & 46052 & 44691 & \\
\hline HIP & & 24740 & 28360 & 3572 & 31173 & 30651 & \\
\hline$V$ & (mag) & 6.14 & 1.89 & 5.65 & 5.82 & 5.55 & \\
\hline$\pi$ & (m.a.s.) & 8.19 & 40.21 & 10.75 & 12.41 & 13.34 & \\
\hline Sp & & $\mathrm{B} 9 \mathrm{~V}+\mathrm{B} 9.6 \mathrm{~V}$ & $\mathrm{~A} 1 \mathrm{Vm}+\mathrm{A} 1 \mathrm{Vm}$ & $\mathrm{A} 2 \mathrm{IV}+\mathrm{F} 2 \mathrm{~V}$ & $\mathrm{~A} 4 \mathrm{~m}+\mathrm{A} 5 \mathrm{~m}$ & $\mathrm{~A} 6 \mathrm{IV}+\mathrm{F} 0 \mathrm{~V}$ & \\
\hline \multicolumn{8}{|c|}{ [Orbital elements] } \\
\hline $\mathrm{JD}(\min \mathrm{I})$ & (day) & 2452501.392 & 2452500.573 & 2454509.297 & 2452501.814 & 2433153.862 & from GCVS \\
\hline$P$ & (day) & 4.134651 & 3.960036 & 4.467222 & 2.52501936 & 9.945079 & from GCVS \\
\hline$K_{1}$ & $\left(\mathrm{~km} \mathrm{~s}^{-1}\right)$ & 107.20 & 107.75 & 73.35 & 116.81 & 66.00 & \\
\hline$K_{2}$ & $\left(\mathrm{~km} \mathrm{~s}^{-1}\right)$ & 115.90 & 111.25 & 125.70 & 126.49 & 84.00 & \\
\hline$\gamma$ & $\left(\mathrm{km} \mathrm{s}^{-1}\right)$ & 25.10 & -17.00 & 8.14 & -8.47 & -12.00 & \\
\hline$e$ & & 0.000 & 0.002 & 0.000 & 0.000 & 0.079 & \\
\hline$i_{\text {orb }}$ & (deg) & 88.52 & 76.91 & 88.30 & 87.55 & 87.45 & \\
\hline \multicolumn{8}{|c|}{ [Stellar parameters] } \\
\hline$M_{1}$ & $\left(M_{\odot}\right)$ & 2.480 & 2.376 & 2.310 & 1.964 & 1.927 & \\
\hline$M_{2}$ & $\left(M_{\odot}\right)$ & 2.294 & 2.291 & 1.350 & 1.814 & 1.507 & \\
\hline$R_{1}$ & $\left(R_{\odot}\right)$ & 1.781 & 2.762 & 2.530 & 1.927 & 2.570 & \\
\hline$R_{2}$ & $\left(R_{\odot}\right)$ & 1.816 & 2.568 & 1.350 & 1.841 & 1.590 & \\
\hline$T_{\text {eff }, 1}$ & $(\mathrm{~K})$ & 10950 & 9350 & 9200 & 7960 & 7570 & \\
\hline$T_{\mathrm{eff}, 2}$ & $(\mathrm{~K})$ & 10350 & 9200 & 6700 & 7670 & 6980 & \\
\hline $\log g_{1}$ & $(\operatorname{dex})$ & 4.331 & 3.932 & 3.995 & 4.162 & 3.900 & \\
\hline $\log g_{2}$ & $(\mathrm{dex})$ & 4.280 & 3.979 & 4.307 & 4.167 & 4.214 & \\
\hline$v_{\mathrm{t}, 1}$ & $\left(\mathrm{~km} \mathrm{~s}^{-1}\right)$ & 1.0 & 2.9 & 3.0 & 4.0 & 3.8 & $v_{\mathrm{t}}\left(T_{\text {eff }}\right)$ formula \\
\hline$v_{\mathrm{t}, 2}$ & $\left(\mathrm{~km} \mathrm{~s}^{-1}\right)$ & 1.6 & 3.0 & 2.6 & 3.9 & 3.1 & $v_{\mathrm{t}}\left(T_{\mathrm{eff}}\right)$ formula \\
\hline$v_{\mathrm{e}} \sin i_{1}$ & $\left(\mathrm{~km} \mathrm{~s}^{-1}\right)$ & 23.2 & 35.9 & 29.3 & 36.2 & 15.2 & O I $7771-5$ fitting \\
\hline$v_{\mathrm{e}} \sin i_{2}$ & $\left(\mathrm{~km} \mathrm{~s}^{-1}\right)$ & 22.6 & 34.1 & 13.2 & 38.8 & 11.6 & O I $7771-5$ fitting \\
\hline
\end{tabular}

The data of $\mathrm{JD}(\min \mathrm{I})$ and $P$ were taken from the web site of General Catalogue of Variable Stars (Samus et al. 2017). The values of $v_{\mathrm{t}, 1}$

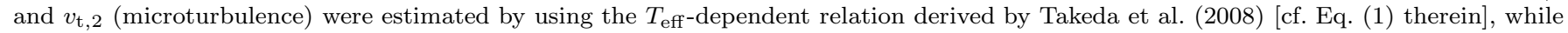
$v_{\mathrm{e}} \sin i_{1}$ and $v_{\mathrm{e}} \sin _{2}$ (projected rotational velocity) are the results of $\mathrm{O}$ I $7771-5$ fitting. All other data were adopted from the compilation of Ecker et al. (2014), which should be consulted for more details (e.g., original references, involved errors, etc.). 
Table 2. Observational dates and radial velocities.

\begin{tabular}{|c|c|c|c|c|c|c|c|c|c|}
\hline $\begin{array}{c}\text { Spectrum label } \\
\text { (1) }\end{array}$ & $\begin{array}{c}\mathrm{JD}(+2400000) \\
(2)\end{array}$ & $\begin{array}{l}\text { Phase } \\
\text { (3) }\end{array}$ & $\begin{array}{r}V_{1}^{\text {hel }} \\
(4)\end{array}$ & $\begin{array}{r}V_{2}^{\text {hel }} \\
(5)\end{array}$ & $\begin{array}{r}\delta V^{\text {hel }} \\
(6)\end{array}$ & $\begin{array}{r}V_{1}^{\text {local }} \\
\quad(7)\end{array}$ & $\begin{array}{r}V_{2}^{\text {local }} \\
(8)\end{array}$ & $V_{1}^{\text {emp }}$ & $\begin{array}{c}V_{2}^{\mathrm{emp}} \\
(10)\end{array}$ \\
\hline araur_20101214A & 55545.010 & 0.125 & -50.6 & 107.0 & -0.6 & -50.1 & 107.6 & -45.1 & 112.6 \\
\hline araur_20101214B & 55545.201 & 0.171 & -69.2 & 127.0 & -1.0 & -68.1 & 128.0 & -63.1 & 133.0 \\
\hline araur_20101215A & 55546.022 & 0.370 & -53.2 & 109.8 & -1.1 & -52.1 & 110.9 & -47.1 & 115.9 \\
\hline araur_20101215B & 55546.272 & 0.430 & -20.5 & 74.4 & -1.7 & -18.8 & 76.1 & -13.8 & 81.1 \\
\hline araur_20101216A & 55546.999 & 0.606 & 91.2 & -46.4 & -1.6 & 92.8 & -44.8 & 97.8 & -39.8 \\
\hline araur_20101218A & 55548.961 & 0.080 & -26.8 & 81.2 & -2.6 & -24.2 & 83.8 & -19.2 & 88.8 \\
\hline araur_20101218B & 55549.163 & 0.129 & -52.7 & 109.2 & -3.0 & -49.6 & 112.2 & -44.6 & 117.2 \\
\hline araur_20101218C & 55549.284 & 0.159 & -64.9 & 122.4 & -3.3 & -61.6 & 125.7 & -56.6 & 130.7 \\
\hline araur_20101220A & 55550.982 & 0.569 & 70.3 & -23.7 & -3.7 & 73.9 & -20.1 & 79.9 & -14.1 \\
\hline araur_20101220B & 55551.152 & 0.610 & 93.6 & -48.9 & -4.0 & 97.6 & -44.9 & 103.6 & -38.9 \\
\hline araur_20101220C & 55551.295 & 0.645 & 109.8 & -66.5 & -4.3 & 114.1 & -62.1 & 120.1 & -56.1 \\
\hline betaur_20101214A & 55545.043 & 0.798 & 86.1 & -122.9 & 3.7 & 82.4 & -126.6 & & \\
\hline betaur_20101214B & 55545.255 & 0.852 & 69.6 & -106.0 & 3.3 & 66.4 & -109.3 & & \\
\hline betaur_20101215A & 55546.087 & 0.062 & -57.8 & 25.2 & 3.1 & -60.9 & 22.1 & & \\
\hline betaur_20101215B & 55546.261 & 0.106 & -83.4 & 51.7 & 2.7 & -86.1 & 49.0 & & \\
\hline betaur_20101216A & 55547.023 & 0.298 & -119.7 & 89.3 & 2.7 & -122.5 & 86.6 & & \\
\hline betaur_20101218A & 55548.984 & 0.793 & 87.0 & -123.9 & 1.8 & 85.2 & -125.7 & & \\
\hline betaur_20101218B & 55549.129 & 0.830 & 77.6 & -114.3 & 1.5 & 76.1 & -115.8 & & \\
\hline betaur_20101218C & 55549.314 & 0.877 & 58.4 & -94.6 & 1.2 & 57.3 & -95.8 & & \\
\hline betaur_20101220A & 55551.005 & 0.304 & -118.5 & 88.0 & 0.8 & -119.3 & 87.3 & & \\
\hline betaur_20101220B & 55551.203 & 0.354 & -102.7 & 71.3 & 0.4 & -103.1 & 70.9 & & \\
\hline betaur_20101220C & 55551.356 & 0.393 & -84.6 & 52.3 & 0.2 & -84.7 & 52.2 & & \\
\hline yzcas_20101214A & 55544.903 & 0.823 & 73.9 & -104.5 & -5.9 & 79.8 & -98.5 & & \\
\hline yzcas_20101214B & 55545.136 & 0.875 & 59.9 & -80.5 & -6.1 & 66.0 & -74.4 & & \\
\hline yzcas_20101216A & 55546.968 & 0.286 & -63.4 & 130.7 & -6.5 & -56.9 & 137.2 & & \\
\hline yzcas_20101218A & 55548.906 & 0.719 & 80.1 & -115.2 & -6.9 & 87.0 & -108.4 & & \\
\hline yzcas_20101218B & 55549.071 & 0.756 & 81.4 & -117.5 & -7.0 & 88.5 & -110.4 & & \\
\hline yzcas_20101218C & 55549.233 & 0.793 & 78.9 & -113.1 & -7.1 & 85.9 & -106.0 & & \\
\hline yzcas_20101220A & 55550.905 & 0.167 & -55.4 & 117.1 & -7.4 & -48.1 & 124.5 & & \\
\hline yzcas_20101220B & 55551.101 & 0.211 & -63.0 & 130.0 & -7.5 & -55.5 & 137.5 & & \\
\hline yzcas_20101220C & 55551.241 & 0.242 & -65.1 & 133.7 & -7.5 & -57.6 & 141.2 & & \\
\hline wwaur_20101214A & 55545.053 & 0.234 & -124.7 & 117.4 & 7.5 & -132.3 & 109.9 & & \\
\hline wwaur_20101214B & 55545.262 & 0.317 & -115.0 & 106.9 & 7.1 & -122.1 & 99.9 & & \\
\hline wwaur_20101215A & 55546.054 & 0.631 & 77.1 & -101.1 & 7.0 & 70.1 & -108.1 & & \\
\hline wwaur_20101215B & 55546.204 & 0.690 & 100.2 & -126.2 & 6.7 & 93.5 & -132.8 & & \\
\hline (wwaur_20101216A) & 55547.028 & 0.017 & -20.7 & 4.7 & 6.6 & $(-27.2)$ & $(-1.8)$ & & \\
\hline wwaur_20101218A & 55548.987 & 0.793 & 104.2 & -130.4 & 5.6 & 98.6 & -136.0 & & \\
\hline wwaur_20101218B & 55549.134 & 0.851 & 85.8 & -110.5 & 5.3 & 80.5 & -115.8 & & \\
\hline wwaur_20101218C & 55549.318 & 0.924 & 45.5 & -66.9 & 4.9 & 40.6 & -71.8 & & \\
\hline wwaur_20101220A & 55551.009 & 0.593 & 56.1 & -78.4 & 4.5 & 51.6 & -83.0 & & \\
\hline wwaur_20101220B & 55551.179 & 0.661 & 90.5 & -115.6 & 4.2 & 86.3 & -119.8 & & \\
\hline wwaur_20101220C & 55551.326 & 0.719 & 106.1 & -132.6 & 3.8 & 102.3 & -136.4 & & \\
\hline (rrlyn_20101214A) & 55545.084 & 0.487 & -27.0 & -18.6 & 5.2 & -32.2 & -23.8 & $(-5.0)$ & $(-30.0)$ \\
\hline rrlyn_20101214B & 55545.292 & 0.508 & -18.8 & -29.1 & 4.9 & -23.7 & -33.9 & 3.0 & -40.0 \\
\hline rrlyn_20101215A & 55546.163 & 0.596 & 17.7 & -65.5 & 4.6 & 13.1 & -70.1 & 29.0 & -74.0 \\
\hline rrlyn_20101215B & 55546.303 & 0.610 & 23.4 & -69.9 & 4.4 & 19.0 & -74.3 & 33.0 & -77.0 \\
\hline rrlyn_20101216A & 55547.051 & 0.685 & 48.9 & -86.1 & 4.4 & 44.5 & -90.4 & 43.0 & -90.0 \\
\hline rrlyn_20101218A & 55549.010 & 0.882 & 35.9 & -64.3 & 3.5 & 32.3 & -67.8 & 21.0 & -62.0 \\
\hline rrlyn_20101218B & 55549.193 & 0.901 & 29.1 & -57.7 & 3.3 & 25.8 & -61.0 & 15.0 & -53.0 \\
\hline rrlyn_20101218C & 55549.368 & 0.918 & 22.2 & -50.8 & 3.0 & 19.2 & -53.8 & 10.0 & -45.0 \\
\hline rrlyn_20101220A & 55551.032 & 0.086 & -43.7 & 33.5 & 2.6 & -46.4 & 30.9 & -55.0 & 39.0 \\
\hline rrlyn_20101220B & 55551.206 & 0.103 & -49.0 & 42.1 & 2.4 & -51.4 & 39.8 & -62.0 & 47.0 \\
\hline rrlyn_20101220C & 55551.361 & 0.119 & -53.4 & 49.4 & 2.1 & -55.5 & 47.2 & -67.0 & 54.0 \\
\hline
\end{tabular}

(1) Spectrum label (e.g., araur_20101218C is the 3rd spectrum of AR Aur observed on 2010 December 18). (2) Heliocentric Julian day. (3) Orbital phase calculated from the $P$ and $\mathrm{JD}(\mathrm{min} \mathrm{I})$ values given in Table 1. (4) Predicted heliocentric radial velocity (in $\mathrm{km} \mathrm{s}^{-1}$ ) for the primary calculated with the orbital elements given in Table 1. (5) Predicted heliocentric radial velocity for the secondary. (6) Heliocentric correction $\left(\mathrm{km} \mathrm{s}^{-1}\right)$ computed by the "rvcorrect" task of IRAF. (7) Predicted local topocentric radial velocity (km s ${ }^{-1}$ ) for the primary. (8) Predicted local topocentric radial velocity $\left(\mathrm{km} \mathrm{s}^{-1}\right)$ for the secondary. (9) Empirically determined radial velocity of the primary directly measured from the spectrum by comparing the simulated double-line profile of O I 7771-5 triplet with the observed one. (10) Empirically determined radial velocity of the secondary. Note that $V_{1}^{\text {emp }}$ and $V_{2}^{\text {emp }}$ were used for spectrum disentangling for AR Aur and RR Lyn, 
Table 3. Adopted luminosity ratio $l_{1}: l_{2}$ at each wavelength region.

\begin{tabular}{ccccc}
\hline \hline Star & $5350-5400 \AA$ & $6130-6180 \AA$ & $7440-7500 \AA$ & $7750-7800 \AA$ \\
\hline AR Aur & $0.520: 0.480$ & $0.518: 0.482$ & $0.515: 0.485$ & $0.515: 0.485$ \\
$\beta$ Aur & $0.545: 0.455$ & $0.544: 0.456$ & $0.543: 0.457$ & $0.543: 0.457$ \\
YZ Cas & $0.917: 0.083$ & $0.903: 0.097$ & $0.883: 0.117$ & $0.879: 0.121$ \\
WW Aur & $0.559: 0.441$ & $0.553: 0.447$ & $0.546: 0.454$ & $0.545: 0.455$ \\
RR Lyn & $0.788: 0.212$ & $0.777: 0.223$ & $0.764: 0.236$ & $0.761: 0.239$ \\
\hline
\end{tabular}

Table 4. Accomplished S/N ratios of the disentangled spectra.

\begin{tabular}{ccccc}
\hline Star & $\sim 5380 \AA$ & $\sim 6150 \AA$ & $\sim 7460 \AA$ & $\sim 7770 \AA$ \\
\hline AR Aur (1) & 400 & 500 & 350 & 400 \\
AR Aur (2) & 400 & 400 & 300 & 350 \\
$\beta$ Aur (1) & 700 & 600 & 450 & 600 \\
$\beta$ Aur (2) & 600 & 600 & 400 & 500 \\
YZ Cas (1) & 600 & 700 & 500 & 600 \\
YZ Cas (2) & 80 & 90 & 70 & 70 \\
WW Aur (1) & 400 & 450 & 350 & 400 \\
WW Aur (2) & 400 & 400 & 300 & 400 \\
RR Lyn (1) & 500 & 600 & 350 & 600 \\
RR Lyn (2) & 150 & 200 & 150 & 200 \\
\hline
\end{tabular}

Table 5. Outline of spectrum-fitting analysis in this study.

\begin{tabular}{ccccc}
\hline \hline Main purpose & Fitting range $(\AA)$ & Abundances varied* & Atomic data source & Figure \\
\hline C abundance from C I 5380 & $5375-5390$ & $\mathrm{C}, \mathrm{Ti}, \mathrm{Fe}$ & KB95m1 & Fig. 4 \\
N abundance from N I 7468 & $7457-7472$ & $\mathrm{~N}, \mathrm{Fe}$ & KB95m2 & Fig. 5 \\
O abundance from O I 6156-8 & $6140-6168$ & $\mathrm{O}, \mathrm{Na}, \mathrm{Si}, \mathrm{Ca}, \mathrm{Fe}, \mathrm{Ba}$ & $\mathrm{KB} 95$ & Fig. 6 \\
$v_{\mathrm{e}} \sin i$ determination from O I 7771-5 & $7765-7785$ & $\mathrm{O}, \mathrm{Fe}$ & KB95m3 & Fig. 7 \\
\hline
\end{tabular}

* The abundances of all other elements than these were fixed in the fitting.

KB95m1 - All the atomic line data presented in Kurucz \& Bell (1995) were used, excepting that the contribution of Fe I 5382.474 $\left(\chi_{\text {low }}=4.371 \mathrm{eV}\right)$ was neglected (because we found its $g f$ value to be erroneously too large).

KB95m2 - All the atomic line data were taken from Kurucz \& Bell (1995), excepting that the contribution of S I $7468.588\left(\chi_{\text {low }}=7.867\right.$ eV) was neglected (because we found its $g f$ value to be erroneously too large).

KB95 - All the atomic line data given in Kurucz \& Bell (1995) were used unchanged.

KB95m3 - All the atomic line data were taken from Kurucz \& Bell (1995), except for the $\log g f$ value of Fe I $7780.552\left(\chi_{\text {low }}=4.473 \mathrm{eV}\right)$, for which we adopted -0.066 (cf. Takeda \& Sadakane 1997). 
Table 6. Adopted atomic data of relevant CNO lines.

\begin{tabular}{ccccccccc}
\hline Line & $\begin{array}{c}\text { Multiplet } \\
\text { No. }\end{array}$ & $\begin{array}{c}\text { Equivalent } \\
\text { Width }\end{array}$ & $\begin{array}{c}\lambda \\
(\AA)\end{array}$ & $\begin{array}{c}\chi_{\text {low }} \\
(\mathrm{eV})\end{array}$ & $\begin{array}{c}\log g f \\
(\mathrm{dex})\end{array}$ & $\begin{array}{c}\text { Gammar } \\
(\mathrm{dex})\end{array}$ & $\begin{array}{c}\text { Gammas } \\
(\mathrm{dex})\end{array}$ & $\begin{array}{c}\text { Gammaw } \\
(\mathrm{dex})\end{array}$ \\
\hline C I 5380 & $(11)$ & $W_{5380}$ & 5380.337 & 7.685 & -1.842 & $(7.89)$ & -4.66 & $(-7.36)$ \\
\hline N I 7468 & $(3)$ & $W_{7468}$ & 7468.312 & 10.336 & -0.270 & 8.64 & -5.40 & $(-7.60)$ \\
\hline O I 6156-8 & $(10)$ & $W_{6156-8}$ & 6155.961 & 10.740 & -1.401 & 7.60 & -3.96 & $(-7.23)$ \\
(9 components) & & & 6155.971 & 10.740 & -1.051 & 7.61 & -3.96 & $(-7.23)$ \\
& & & 6155.989 & 10.740 & -1.161 & 7.61 & -3.96 & $(-7.23)$ \\
& & & 6156.737 & 10.740 & -1.521 & 7.61 & -3.96 & $(-7.23)$ \\
& & & 6156.755 & 10.740 & -0.931 & 7.61 & -3.96 & $(-7.23)$ \\
& & & 6156.778 & 10.740 & -0.731 & 7.62 & -3.96 & $(-7.23)$ \\
& & & 6158.149 & 10.741 & -1.891 & 7.62 & -3.96 & $(-7.23)$ \\
& & & 6158.172 & 10.741 & -1.031 & 7.62 & -3.96 & $(-7.23)$ \\
& & & 6158.187 & 10.741 & -0.441 & 7.61 & -3.96 & $(-7.23)$ \\
\hline O I 7771-5 & $(1)$ & $W_{7771-5}$ & 7771.944 & 9.146 & +0.324 & 7.52 & -5.55 & $(-7.65)$ \\
& & & 7774.166 & 9.146 & +0.174 & 7.52 & -5.55 & $(-7.65)$ \\
& & & 7775.388 & 9.146 & -0.046 & 7.52 & -5.55 & $(-7.65)$ \\
\hline
\end{tabular}

Following columns 3-5 (laboratory wavelength, lower excitation potential, and $\log g f$ value), three kinds of damping parameters are presented in columns 6-8: Gammar is the radiation damping width $\left(\mathrm{s}^{-1}\right)\left[\log \gamma_{\mathrm{rad}}\right]$, Gammas is the Stark damping width ( $\left.\mathrm{s}^{-1}\right)$ per electron density $\left(\mathrm{cm}^{-3}\right)$ at $10^{4} \mathrm{~K}\left[\log \left(\gamma_{\mathrm{e}} / N_{\mathrm{e}}\right)\right]$, and Gammaw is the van der Waals damping width $\left(\mathrm{s}^{-1}\right)$ per hydrogen density $\left(\mathrm{cm}^{-3}\right)$ at $10^{4} \mathrm{~K}$ $\left[\log \left(\gamma_{\mathrm{w}} / N_{\mathrm{H}}\right)\right]$.

All the data were taken from Kurucz \& Bell (1995), except for the parenthesized damping parameters (unavailable in their compilation), for which the default values computed by Kurucz's (1993) WIDTH9 program were assigned.

Table 7. Derived abundances of C, N, O, Na, Si, Ca, Ti, Fe, and Ba.

\begin{tabular}{|c|c|c|c|c|c|c|c|c|c|}
\hline $\begin{array}{c}\text { Star } \\
(1)\end{array}$ & $\begin{array}{l}{[\mathrm{C}]} \\
(2)\end{array}$ & $\begin{array}{l}{[\mathrm{N}]} \\
(3)\end{array}$ & $\begin{array}{l}{[\mathrm{O}]} \\
(4)\end{array}$ & $\begin{array}{c}{[\mathrm{Na}]} \\
(5)\end{array}$ & $\begin{array}{l}{[\mathrm{Si}]} \\
(6)\end{array}$ & $\begin{array}{c}{[\mathrm{Ca}]} \\
(7)\end{array}$ & $\begin{array}{c}{[\mathrm{Ti}]} \\
(8)\end{array}$ & $\begin{array}{c}{[\mathrm{Fe}]} \\
(9)\end{array}$ & $\begin{array}{l}{[\mathrm{Ba}]} \\
(10)\end{array}$ \\
\hline AR Aur (1) & $-1.11:$ & $(<-1.9)$ & -0.43 & +0.30 & +0.25 & +0.34 & +0.51 & +0.47 & -0.05 \\
\hline AR Aur (2) & -0.68 & -0.55 & -0.31 & $\ldots$ & -0.65 & -0.24 & -0.10 & +0.07 & +0.79 \\
\hline$\beta$ Aur (1) & -0.59 & -0.68 & -0.48 & +0.44 & +0.07 & -0.01 & +0.12 & +0.32 & +1.18 \\
\hline$\beta$ Aur (2) & -0.65 & -0.71 & -0.46 & +0.43 & +0.07 & -0.06 & +0.14 & +0.37 & +1.26 \\
\hline YZ Cas (1) & -0.59 & -0.82 & -0.43 & +0.45 & +0.16 & -0.05 & +0.23 & +0.40 & +1.43 \\
\hline YZ Cas (2) & -0.15 & -0.11 & -0.23 & -0.21 & -0.07 & -0.23 & -0.13 & -0.19 & -0.44 \\
\hline WW Aur (1) & -0.90 & -1.00 & -0.34 & +0.04 & -0.12 & -1.05 & -0.09 & +0.09 & +0.97 \\
\hline WW Aur (2) & -0.80 & -0.85 & -0.32 & -0.02 & -0.06 & -1.08 & -0.04 & +0.15 & +1.11 \\
\hline RR Lyn (1) & -0.99 & -1.08 & -0.72 & +0.00 & -0.12 & -0.80 & -0.22 & +0.11 & +1.21 \\
\hline RR Lyn (2) & -0.15 & -0.20 & +0.00 & -0.38 & -0.33 & -0.30 & -0.21 & -0.41 & -0.14 \\
\hline
\end{tabular}

All the results given here are the differential abundances relative to Procyon (see ReadMe of the online material for the detailed reference abundances of Procyon determined in the same manner).

(2) Non-LTE C abundance from C i 5380. (3) Non-LTE N abundance from N i 7468. (4) Non-LTE O abundance from O i 6156-8. (5) LTE $\mathrm{Na}$ abundance derived from 6140-6168 Å fitting. (6) LTE Si abundance derived from 6140-6168 Å fitting. (7) LTE Ca abundance derived

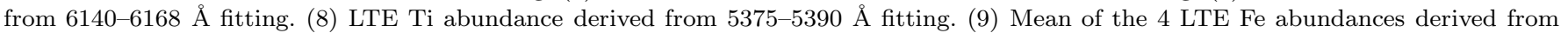

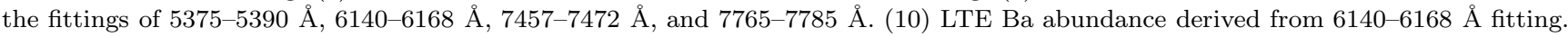
Regarding the data given for the primary of AR Aur, the parenthesized value for $\mathrm{N}$ is the upper limit, while the value for $\mathrm{C}$ with colon (:) is considered to suffer a large uncertainty. 

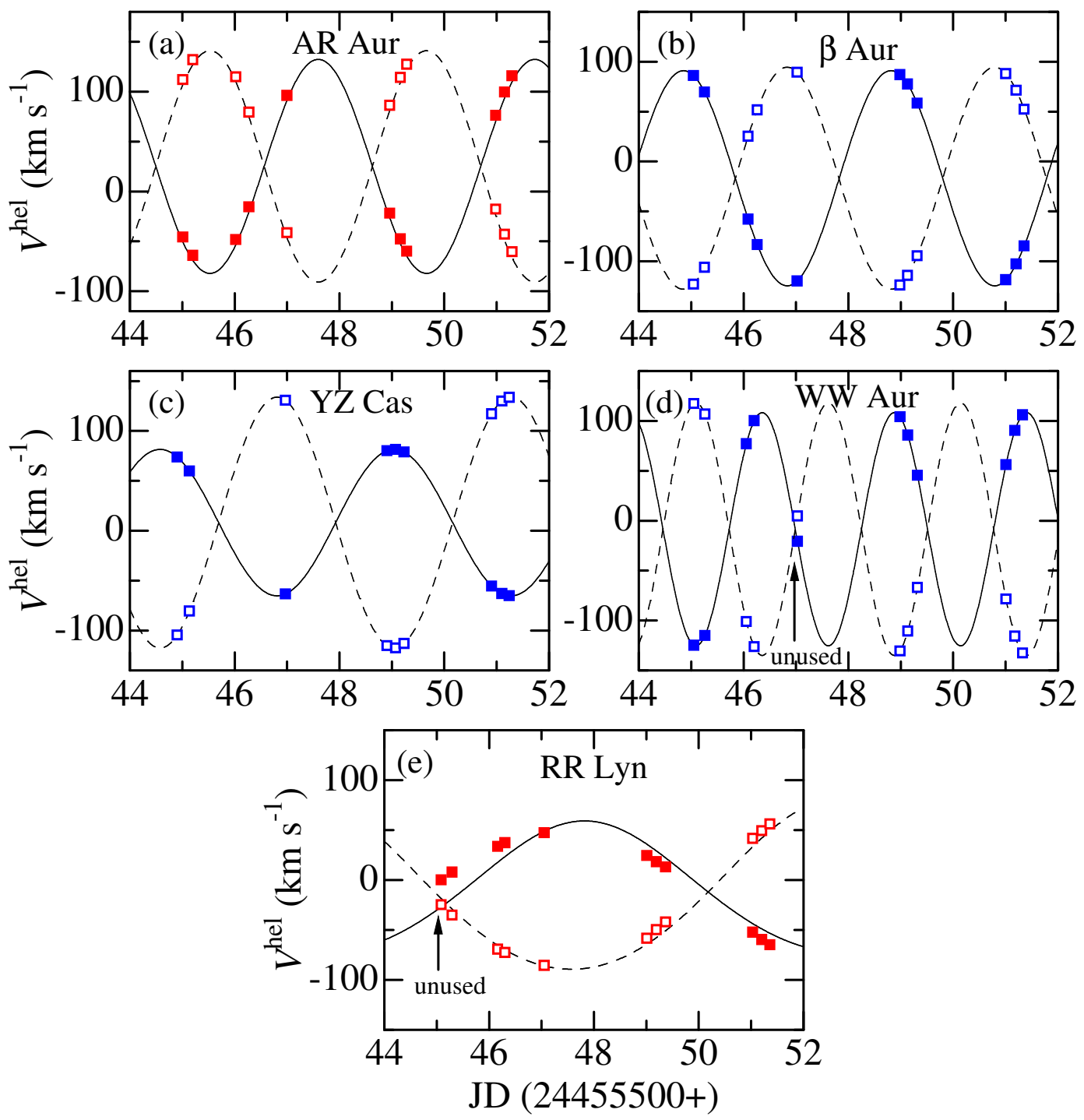

Figure 1. The solid (primary) and dashed (secondary) lines show the predicted heliocentric radial velocity curves calculated by using the orbital elements given in Table 1. The filled (primary) and open (secondary) symbols indicate the radial velocities corresponding to each of the spectra, which were actually used for spectrum disentangling (reduced to the heliocentric system). That is, according to the notation of Table 2, $V_{1}^{\text {local }}+\delta V^{\text {hel }}$ and $V_{2}^{\text {local }}+\delta V^{\text {hel }}$ (for $\beta$ Aur, YZ Cas, and WW Aur), or $V_{1}^{\text {emp }}+\delta V^{\text {hel }}$ and $V_{2}^{\text {emp }}+\delta V^{\text {hel }}$ (for AR Aur and RR Lyn). The data indicated by arrows in panels (d) and (e) were not used, because they correspond to eclipse phases. (a) AR Aur, (b) $\beta$ Aur, (c) YZ Cas, (d) WW Aur, and (e) RR Lyn. 


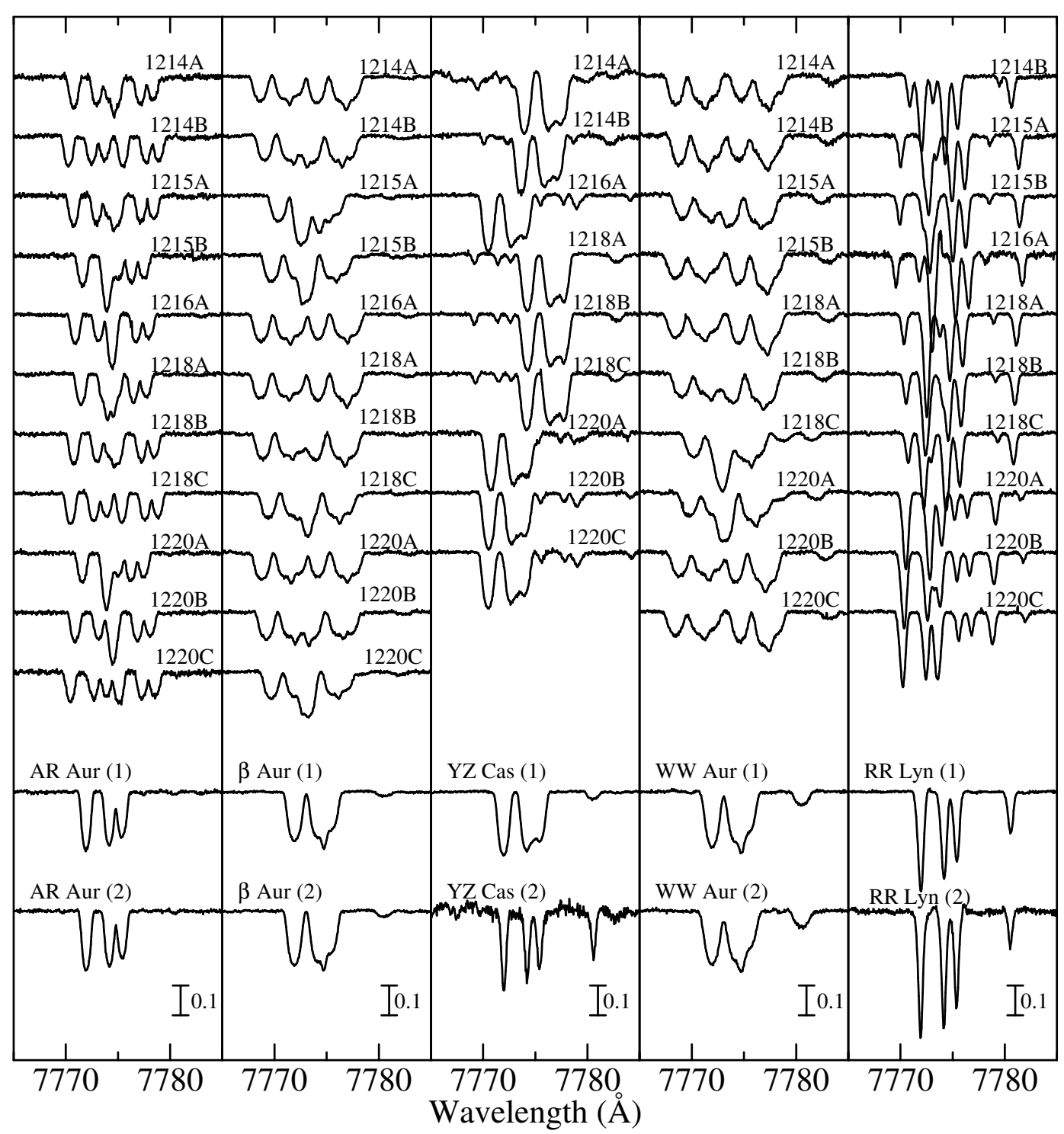

Figure 2. In each of the panels (from left to right: AR Aur, $\beta$ Aur, YZ Cas, WW Aur, and RR Lyn) are shown the original set of spectra used for spectrum entangling (indicated by the observed date and sequence) as well as the resulting disentangled spectra (the lowest two) in the neighborhood of the O I $7771-5$ triplet lines. 


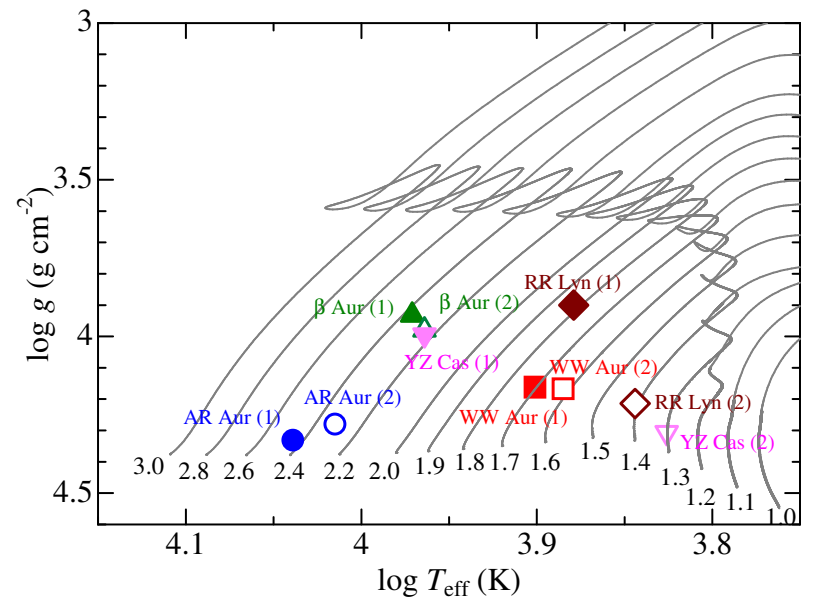

Figure 3. Our program stars plotted on the $T_{\text {eff }}$ vs. $\log g$ diagram. Each of the 5 binary systems are shown by different symbol shapes [circles (AR Aur), triangles ( $\beta$ Aur), inverse triangles (YZ Cas), squares (WW Aur), and diamonds (RR Lyn)], while the filled and open symbols correspond to the primary (1) and the secondary (2) components, respectively. The solid lines show the theoretical relations predicted from PARSEC stellar evolution calculations for solar-metallicity models (Bressan et al. 2012), where the corresponding stellar mass (in unit of $M_{\odot}$ ) is indicated for each track. 

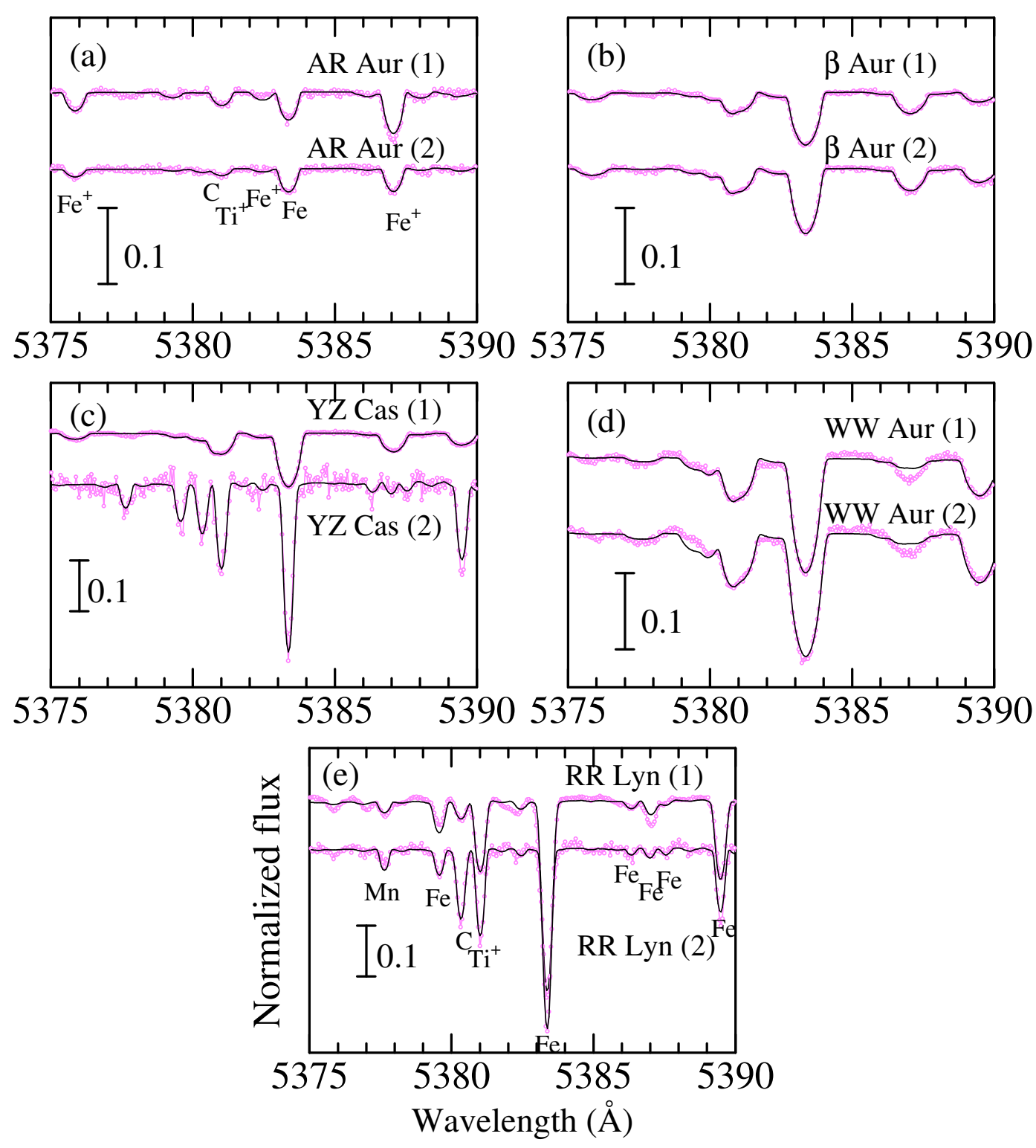

Figure 4. Synthetic spectrum fitting in the 5375-5390 ̊ region comprising the C I 5380 line (along with the lines of $\mathrm{Ti}$ and $\mathrm{Fe}$ ). The best-fit theoretical spectra are shown by black solid lines. while the disentangled observed spectra are plotted by pink symbols. In each panel are shown the results for the primary (upper) as well as the secondary (lower). (a) AR Aur, (b) $\beta$ Aur, (c) YZ Cas, (d) WW Aur, and (e) RR Lyn. 

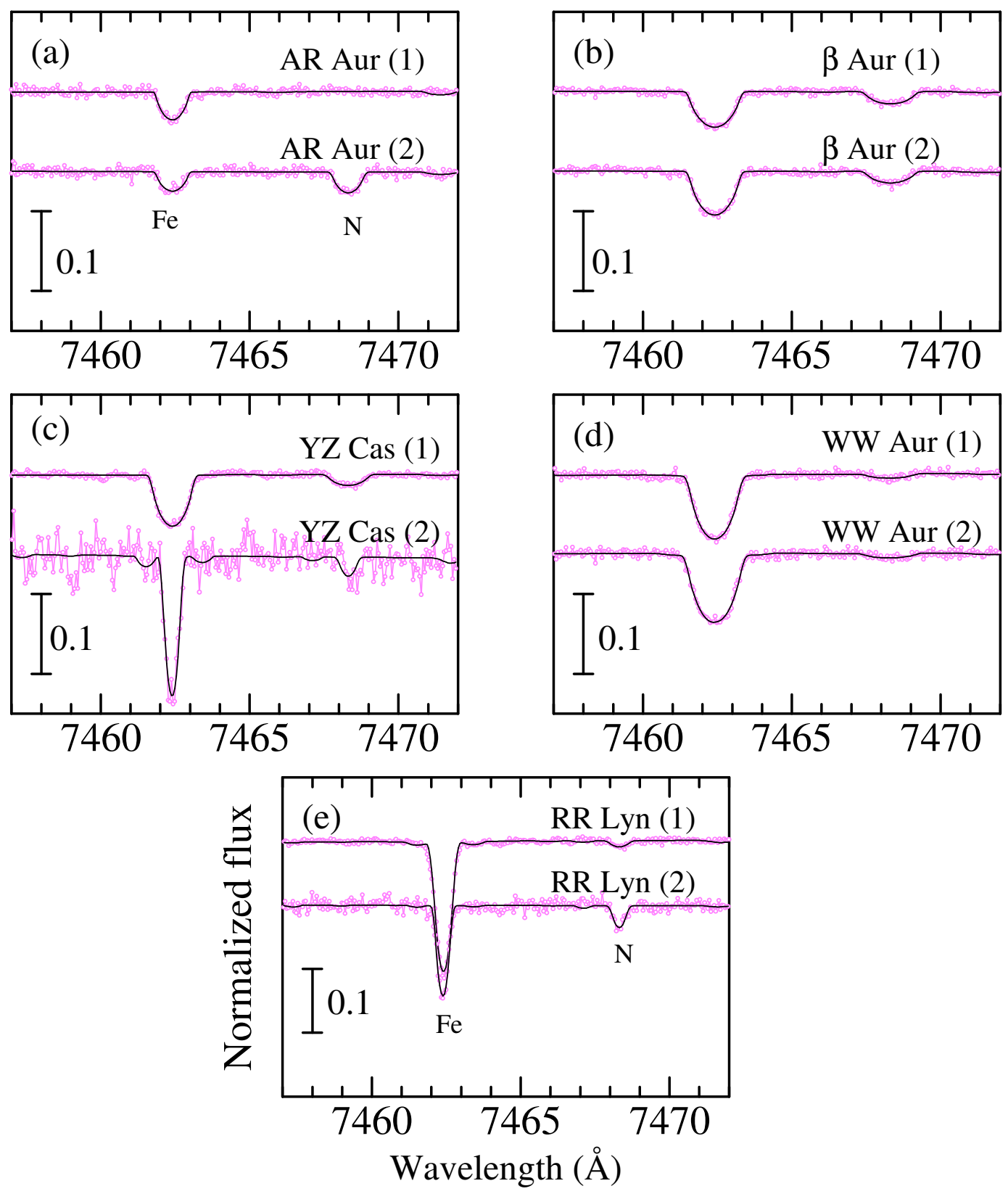

Figure 5. Synthetic spectrum fitting in the 7457-7472 A region comprising the N I 7468 line (along with the line of $\mathrm{Fe}$ ). Otherwise, the same as in Fig. 4. 


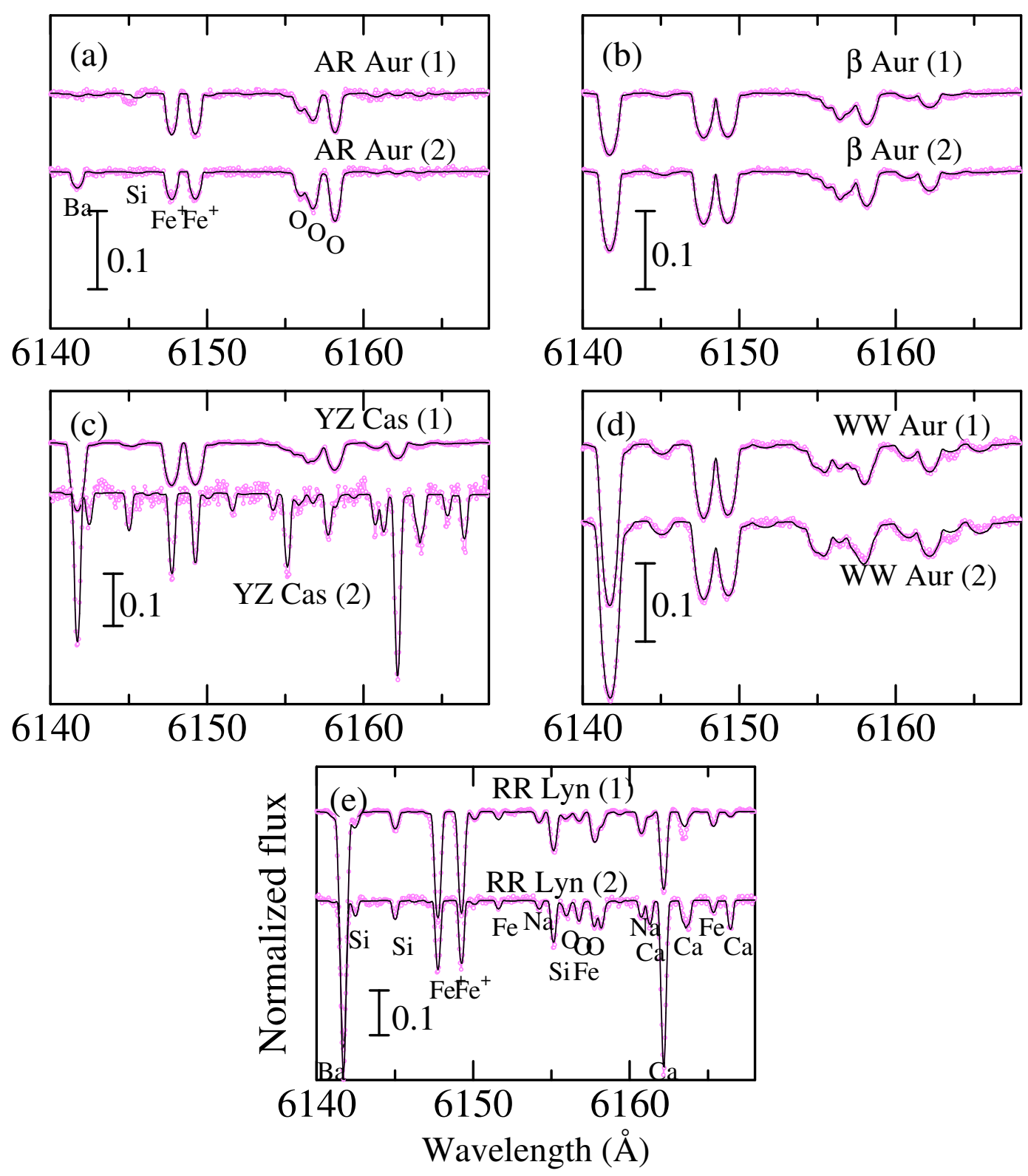

Figure 6. Synthetic spectrum fitting in the 6140-6168 $\AA$ region comprising the O I $6156-8$ lines (along with the lines of $\mathrm{Na}, \mathrm{Si}, \mathrm{Ca}, \mathrm{Fe}$, and $\mathrm{Ba}$ ). Otherwise, the same as in Fig. 4. 

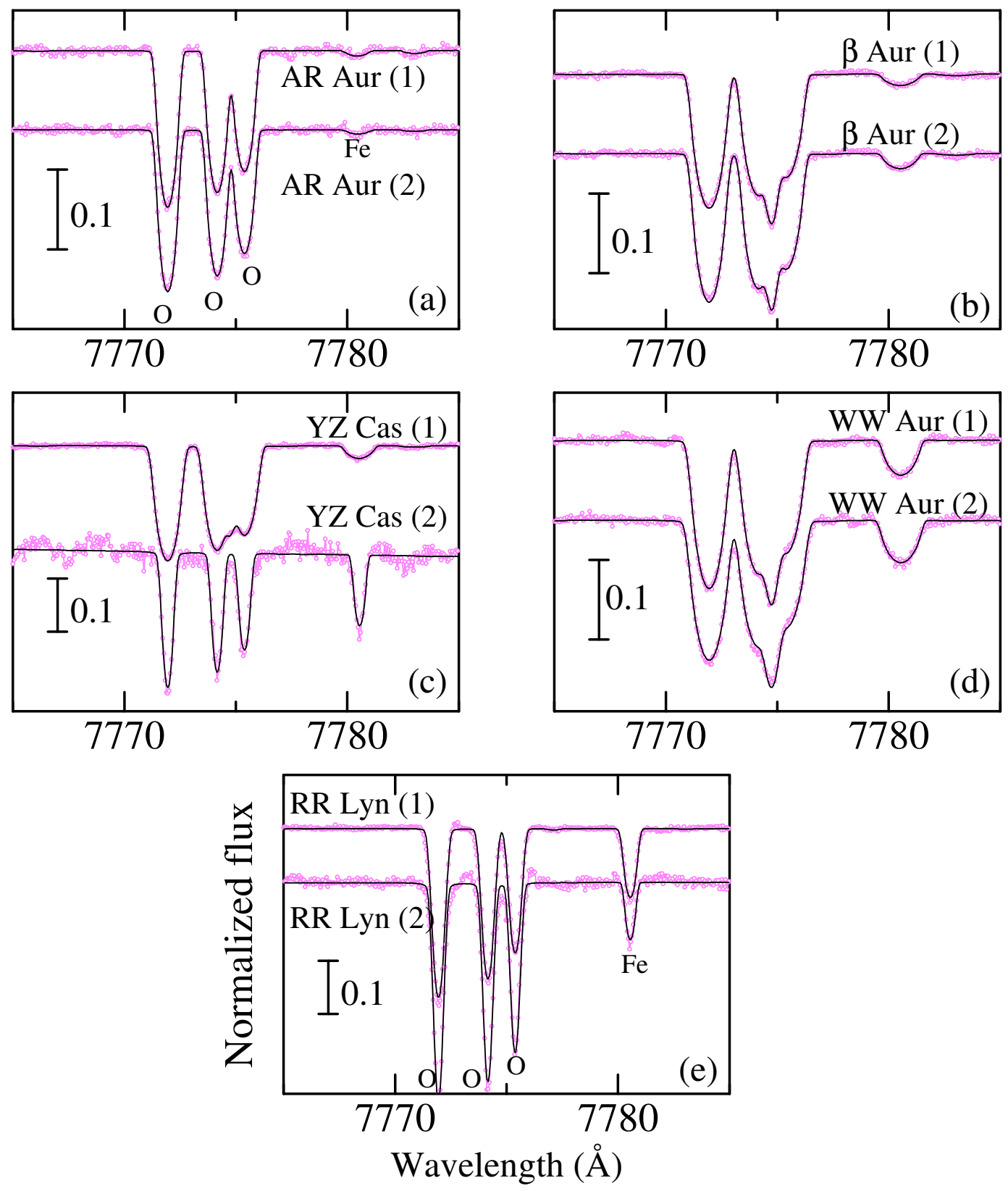

Figure 7. Synthetic spectrum fitting in the 7765-7785 $\AA$ region comprising the O I $7771-5$ lines (along with the line of $\mathrm{Fe})$. Otherwise, the same as in Fig. 4. 

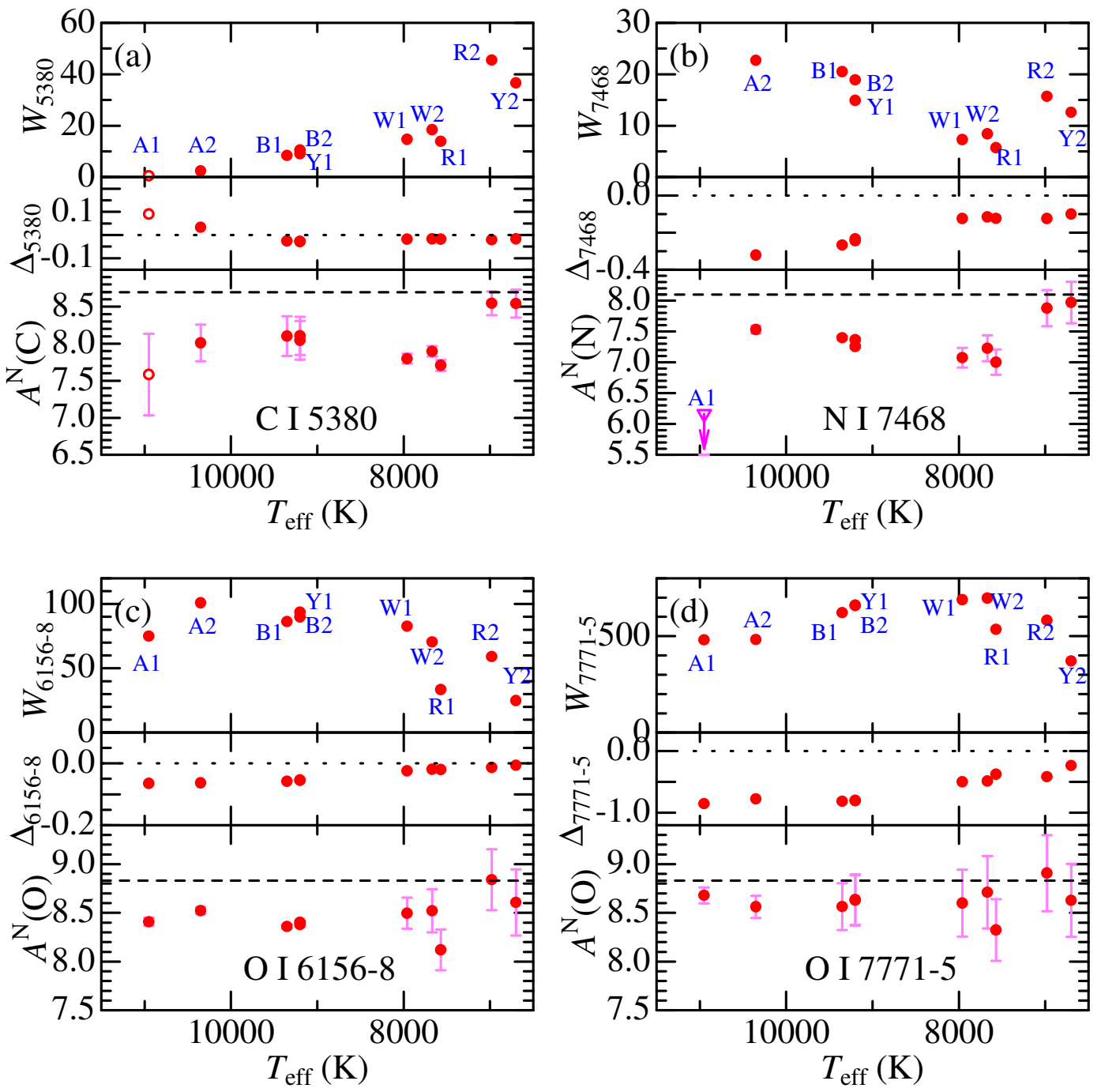

Figure 8. (a) C I 5380-related quantities plotted against $T_{\text {eff }}: W_{5380}$ (equivalent width), $\Delta_{5380}$ (non-LTE correction), and $A^{\mathrm{N}}(\mathrm{C})$ (non-LTE logarithmic number abundance of $\mathrm{C}$ expressed in the usual normalization of $\left.A_{\mathrm{H}}=12\right)$. The error bars in $A^{\mathrm{N}}(\mathrm{C})$ are the combined uncertainties due to those of atmospheric parameters and photometric errors (cf. Sect. 4.3 in Paper I). The codes attached to the symbols are almost self-explanatory; e.g., A1 is AR Aur (1), B2 is $\beta$ Aur (2), or Y2 is YZ Cas (2). The reference $A^{\mathrm{N}}(\mathrm{C})$ value for the standard star Procyon is shown by the horizontal dashed line. (b) Similar to panel (a) but for N I 7460 . (c) Similar to panel (a) but for O I 6156-8. (d) Similar to panel (a) but for O I 7771-5. Note that the open symbols in panel (a) indicate the results with considerably large uncertainties and the downward arrow in panel (b) means the upper limit, which correspond to C and N abundances for AR Aur (1), respectively. 

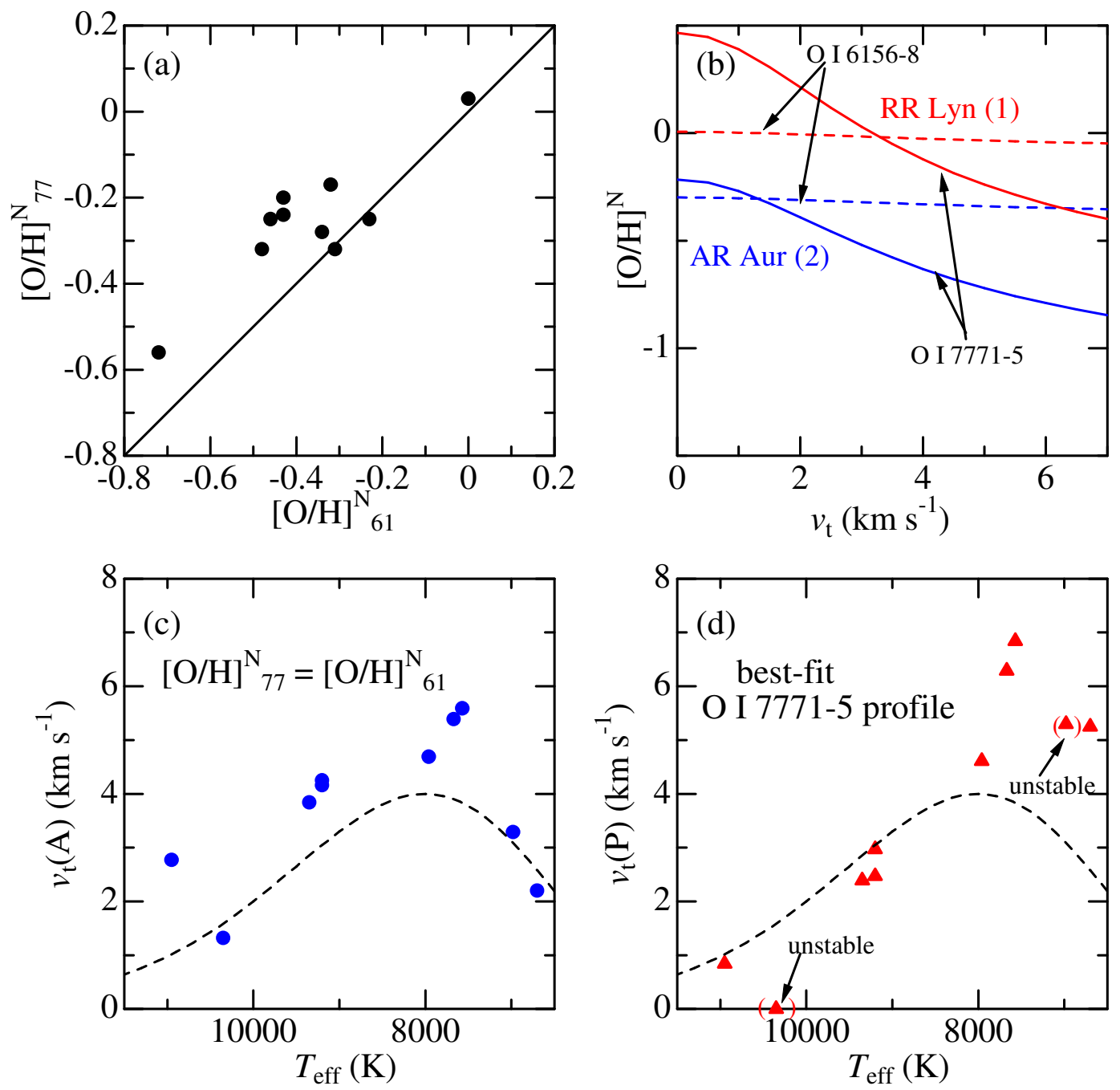

Figure 9. (a) Comparison of two kinds of non-LTE oxygen abundances (relative to Procyon) derived from O I $7771-5$ and $\mathrm{O}$ I $6156-8$ lines. (b) Examples of how $v_{\mathrm{t}}(\mathrm{A})$ (microturbulence derived by requiring the abundance consistency between $[\mathrm{O} / \mathrm{H}]_{77}^{\mathrm{N}}$ and $[\mathrm{O} / \mathrm{H}]_{61}^{\mathrm{N}}$ ) can be determined, shown for the cases of AR Aur (2) and RR Lyn (1). (c) Such determined "abundance-matched" $v_{\mathrm{t}}(\mathrm{A})$ results plotted against $T_{\text {eff }}$. (d) The values of $v_{\mathrm{t}}(\mathrm{P})$ ("profile-based" microturbulence derived from the profile-fitting of O I 7771-5 lines) plotted against $T_{\text {eff }}$. Since the $v_{\mathrm{t}}(\mathrm{P})$ solutions for AR Aur (2) and RR Lyn (2) did not converge showing oscillatory behaviors, only the rough (mean) values are shown only for reference, as indicated by parentheses. The dashed line shown in panels (c) and (d) is the $v_{\mathrm{t}}$ vs. $T_{\text {eff }}$ relation adopted in this study. 

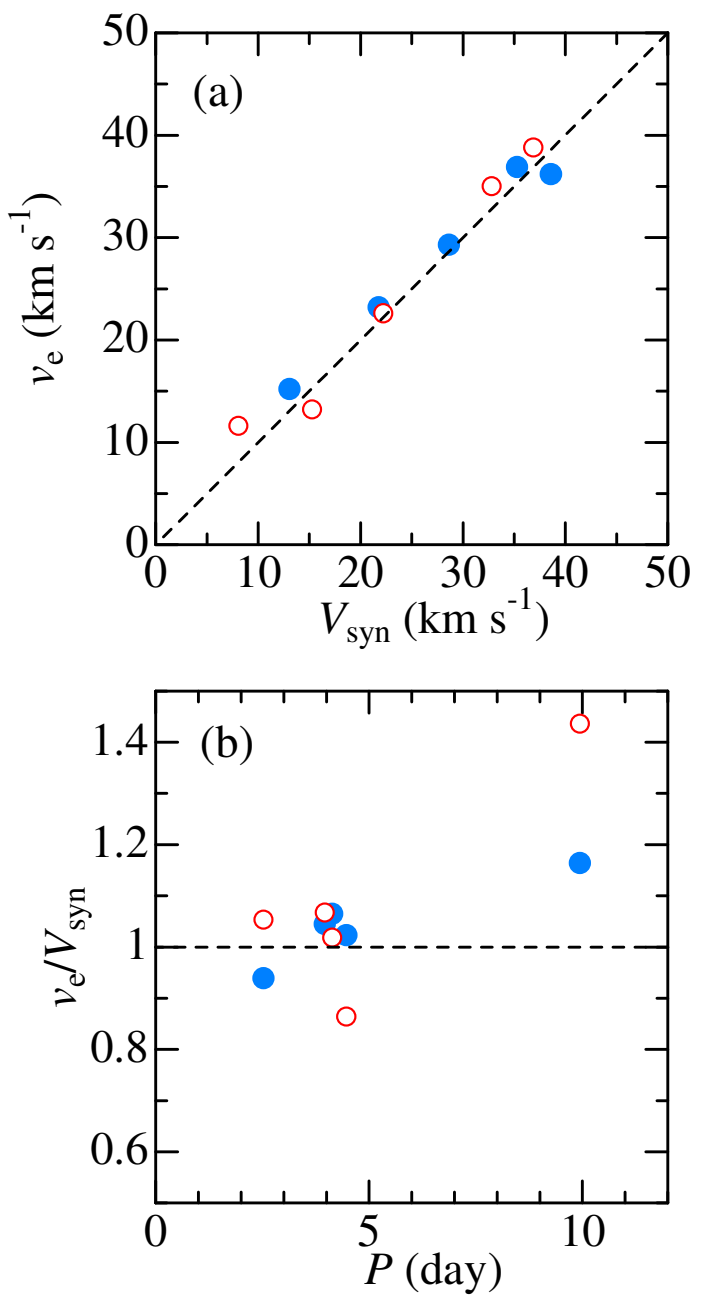

Figure 10. (a) Correlation between the observed equatorial rotational velocity $\left[v_{\mathrm{e}} \equiv\left(v_{\mathrm{e}} \sin i\right) / \sin i_{\mathrm{orb}}\right.$ : assuming the alignment of orbital and rotational axes] and the predicted rotational velocity based on the assumption of synchronization $\left.\left[V_{\mathrm{syn}} \equiv 2 \pi R / P\right)\right]$. The primary and secondary components are denoted by filled and open symbols, respectively. (b) $v_{\mathrm{e}} / V_{\mathrm{syn}}$ ratios plotted against $P$ (period of orbital motion). 

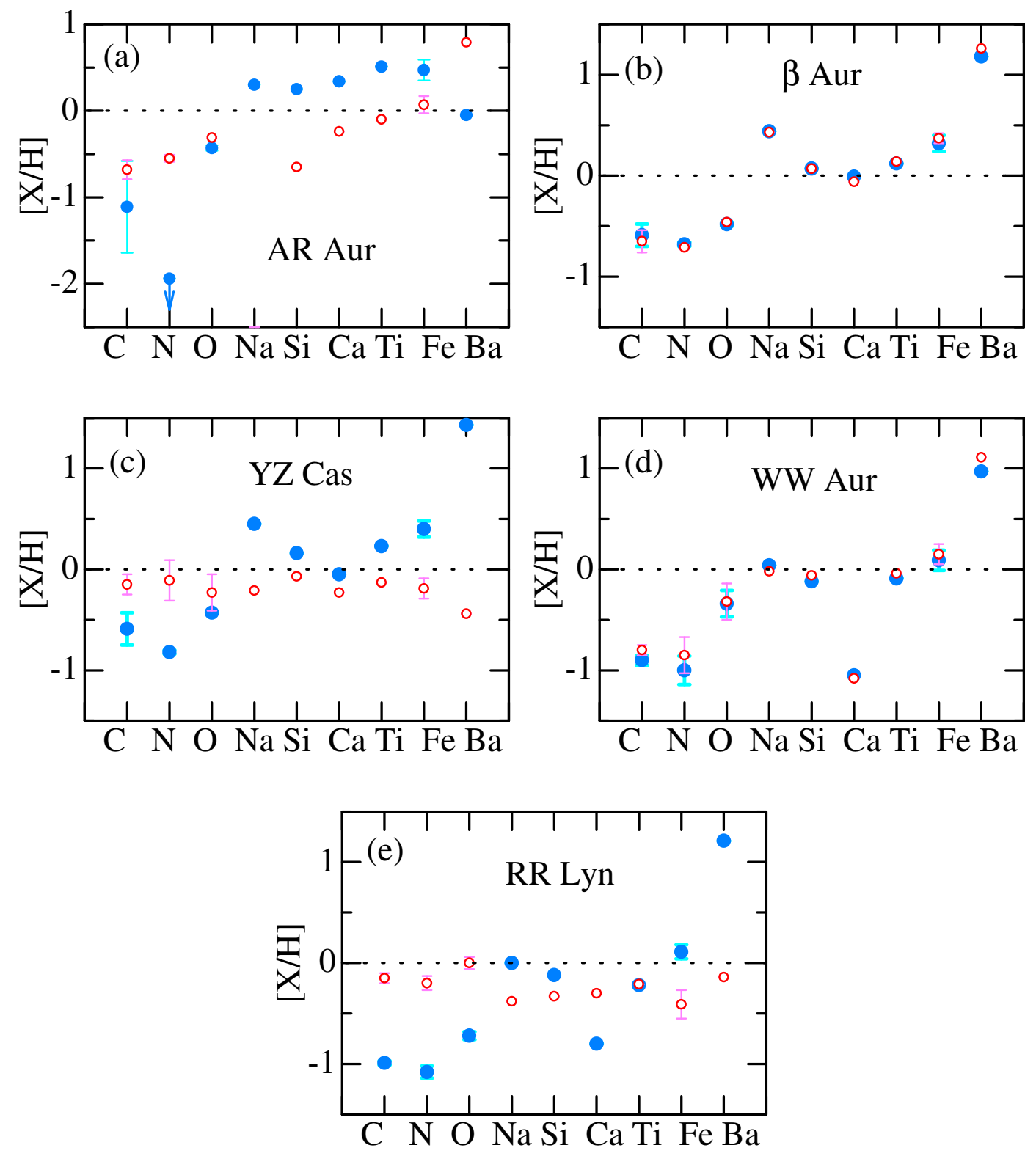

Figure 11. Graphical plots of the $[\mathrm{X} / \mathrm{H}]$ values given in Table 7 . The primary and secondary components are denoted by filled and open symbols, respectively. The attached error bars to $[\mathrm{C} / \mathrm{H}],[\mathrm{N} / \mathrm{H}]$ and $[\mathrm{O} / \mathrm{H}]$ are the same as the case for $A^{\mathrm{N}}$ in Fig. 8, while that of $[\mathrm{Fe} / \mathrm{H}]$ is the standard deviation of the four $[\mathrm{Fe} / \mathrm{H}]$ values (cf. the caption in Table 7). (a) AR Aur, (b) $\beta$ Aur, (c) YZ Cas, (d) WW Aur, and (e) RR Lyn. 

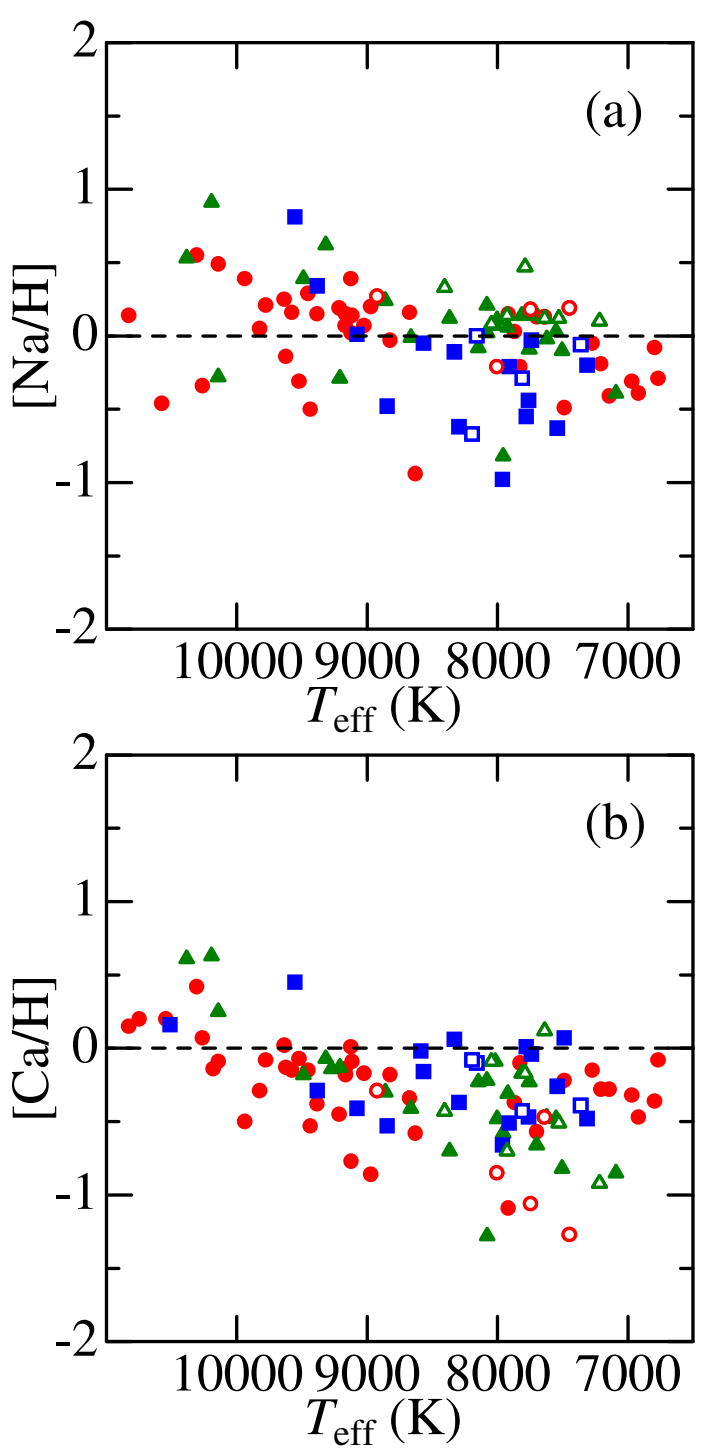

Figure 12. (a) $[\mathrm{Na} / \mathrm{H}]$ vs. $T_{\text {eff }}$ and (b) $[\mathrm{Ca} / \mathrm{H}]$ vs. $T_{\text {eff }}$ relations for $100 \mathrm{~A}$-type main-sequence stars studied in Paper I (these abundance data are given in "paperI_suppl.dat" of the online material). Corresponding $v_{\mathrm{e}} \sin i$ ranges are discriminated by the types of symbols: circles $\left(0<v_{\mathrm{e}} \sin i<30 \mathrm{~km} \mathrm{~s}^{-1}\right)$, triangles $\left(30 \leqslant v_{\mathrm{e}} \sin i<70 \mathrm{~km} \mathrm{~s}^{-1}\right)$, and squares $\left(70 \leqslant v_{\mathrm{e}} \sin i<100 \mathrm{~km} \mathrm{~s}^{-1}\right)$. Open symbols denote 16 stars belonging to the Hyades cluster. 

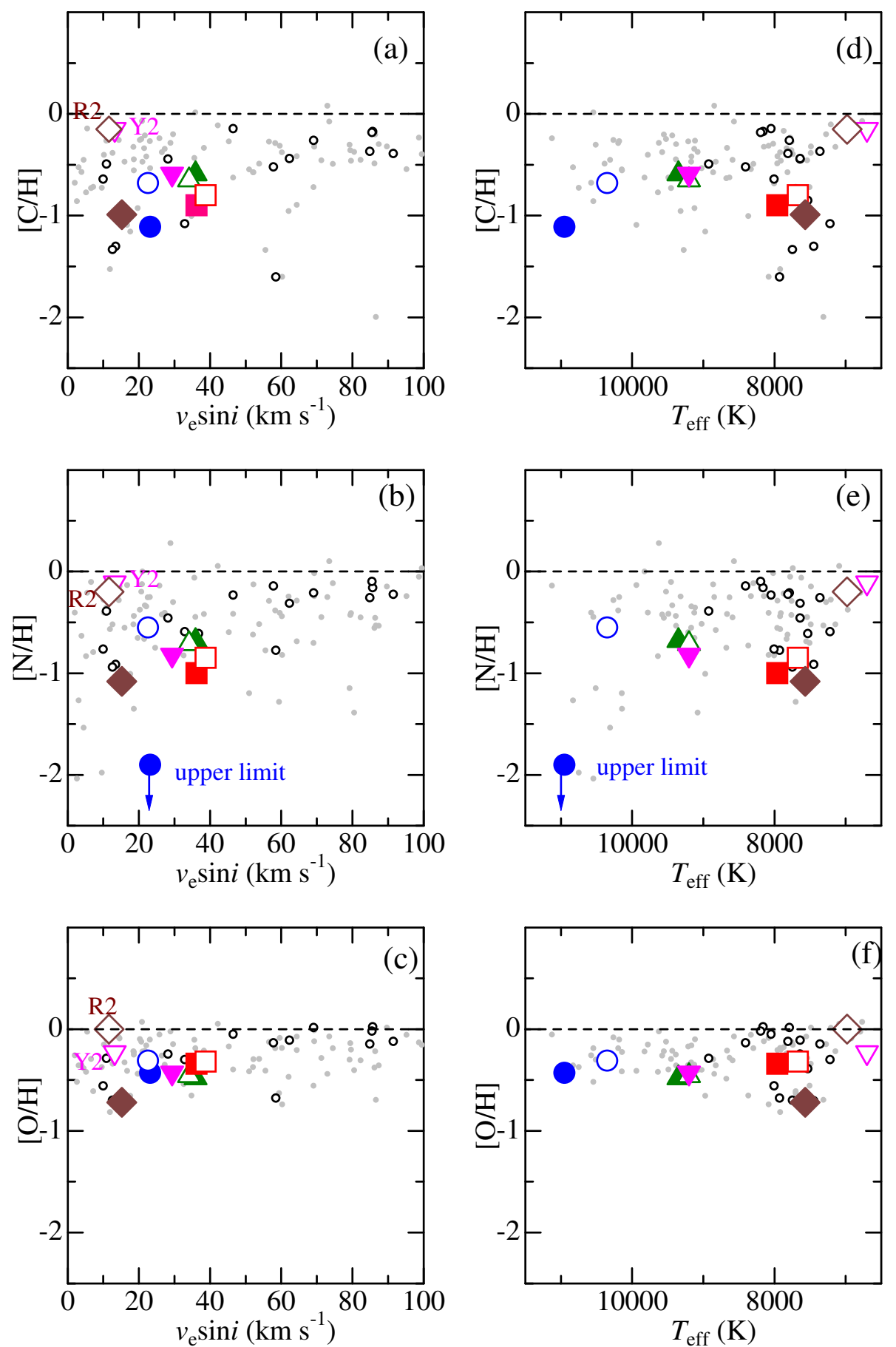

Figure 13. Comparison of the CNO abundances of the 10 program stars (large symbols; the same meaning as in Fig. 3) with those of 100 A-type stars derived in Paper 1 (small symbols; where Hyades stars are denoted by small open circles, while others are by gray dots). In the left $(\mathrm{a}-\mathrm{c})$ and right $(\mathrm{d}-\mathrm{f})$ panels are plotted the results against $v_{\mathrm{e}} \sin i$ and $T_{\mathrm{eff}}$, while the top (a,d), middle (b, e), and bottom (c, f) panels correspond to $[\mathrm{C} / \mathrm{H}],[\mathrm{N} / \mathrm{H}]$, and $[\mathrm{O} / \mathrm{H}]$, respectively. In the left panels (a)-(c), RR Lyn (2) and YZ Cas (2) are denoted as R2 and Y2, in order to remark that these two are normal abundance stars. 